FTUV-10-0126, IFIC/10-03

PNUTP-10-A03

\title{
The degenerate gravitino scenario
}

\author{
Lotfi Boubekeur ${ }^{\mathrm{a}, \mathrm{b}}$, Ki Young Choi ${ }^{\mathrm{c}}$, Roberto Ruiz de Austri ${ }^{\mathrm{b}}$, and Oscar Vives ${ }^{\mathrm{a}, \mathrm{b}}$ \\ a Departament de Física Teòrica, Universitat de València, E-46100, Burjassot, Spain. \\ b Instituto de Física Corpuscular (IFIC), Universitat de València-CSIC, \\ Edificio de Institutos de Paterna, Apt. 22085, E-46071, Valencia, Spain. \\ ${ }^{c}$ Department of Physics, Pusan National University, Busan 609-735, Korea.
}

\begin{abstract}
In this work, we explore the "degenerate gravitino" scenario where the mass difference between the gravitino and the lightest MSSM particle is much smaller than the gravitino mass itself. In this case, the energy released in the decay of the next to lightest sypersymmetric particle (NLSP) is reduced. Consequently the cosmological and astrophysical constraints on the gravitino abundance, and hence on the reheating temperature, become softer than in the usual case. On the other hand, such small mass splittings generically imply a much longer lifetime for the NLSP. We find that, in the constrained MSSM (CMSSM), for neutralino LSP or NLSP, reheating temperatures compatible with thermal leptogenesis are reached for small splittings of order $10^{-2} \mathrm{GeV}$. While for stau NLSP, temperatures of $T_{\mathrm{RH}} \simeq 4 \times 10^{9} \mathrm{GeV}$ can be obtained even for splittings of order of tens of GeVs. This "degenerate gravitino" scenario offers a possible way out to the gravitino problem for thermal leptogenesis in supersymmetric theories.
\end{abstract}

\section{Introduction}

The existence of long-lived massive particles is always welcome in theories beyond the Standard Model (SM) if their lifetime is longer than the age of the universe (or completely stable) as they provide a candidate for the cold Dark Matter (DM) component of the universe to account for the abundance, $\Omega_{\mathrm{CDM}} h^{2} \simeq 0.11$, inferred by cosmological observations. However, long-lived particles decaying after Big Bang Nucleosynthesis (BBN) place the theory in a very difficult situation when confronted with cosmological and astrophysical observation.1 The very successful predictions of standard BBN are spoiled by the energetic products of the decay that can dissociate the produced light elements for lifetimes from $10^{2}$ to $10^{10}$ seconds. If the lifetime is between $10^{10}$ and $10^{13}$

\footnotetext{
${ }^{1}$ Decay products of particles with lifetimes as long as $10^{27}$ sec., see e.g. [1, 2, 3, can still produce observable signatures at present. Particles with longer lifetimes are only constrained from the measured relic density.
} 
seconds, very stringent constraints come from the shape of the Cosmic Microwave Background (CMB) spectrum. For longer lifetimes, the emitted photons can reach us today and be observed as part of the Diffuse Extragalactic Background RAdiation (DEBRA). These observations restrict strongly the released energy in the decays of the long-lived particle at different times and hence its abundance, mass and lifetime.

The paradigm of such long-lived particle conflicting with cosmological and astrophysical observations is the gravitino in supersymmetric theories. The gravitino is the spin $3 / 2$ supersymmetric partner of the graviton and the couplings of the gravitino to ordinary matter are gravitational couplings suppressed by the Planck mass, $M_{\mathrm{Pl}}=\left(8 \pi G_{N}\right)^{-1 / 2} \simeq 2.4 \times 10^{18} \mathrm{GeV}$. Such small couplings make the typical gravitino lifetime (with electroweak scale masses and neglecting the masses of the decay products) of the order of $10^{8}$ seconds. Therefore, they decay after BBN and the gravitino abundance at BBN is severely constrained [4. This has very important consequences in the phenomenology of this model. In fact, these BBN constraints forbid reheating temperatures larger than $10^{6}$ or $10^{7} \mathrm{GeV}$, which precludes thermal leptogenesis from generating a large enough baryon asymmetry.

It is interesting to check whether it is possible to evade these stringent bounds. However, given that the gravitino couplings are completely fixed by supergravity, it is very difficult to change the gravitino production or its decay. The only "free" parameter available (from an effective theory point of view) is the gravitino mass itself and similarly the masses of the decay products. The gravitino gets a mass after supersymmetry breaking and the different soft-breaking terms receive a contribution proportional to the gravitino mass. If supergravity is the mechanism of mediation of the supersymmetry breaking from the hidden sector to the visible sector, we can expect the SUSY masses and the gravitino to be of the same orden2. However, most phenomenological analyses of BBN constraints assume that the released energy in the thermal plasma is of the same order as the gravitino mass itself. Although this is correct in most of the cases where the mass difference between the gravitino and the SUSY particles (typically the lightest MSSM supersymmetric particle (MLSP)) is sizeable, we can ask what happens if the gravitino and the MSSM LSP masses are much closer, $\Delta M=m_{3 / 2}-m_{\mathrm{MLSP}} \ll m_{3 / 2}$. This has two different consequences, first it is evident that the released energy in the thermal plasma is much smaller and this can help to relax the previous bounds. On the other hand, this small mass difference increases the gravitino lifetime and other constraints as the CMB spectrum or diffuse gamma rays come into play.

In this work, we will analyze this scenario that we call "degenerate gravitino" scenario, including both the case where the gravitino is the NLSP decaying to the MSSM LSP and the case where the gravitino itself is the LSP and all SUSY particles decay into the gravitino. In the following, we define $\delta$ as the degree of degeneracy between NLSP and LSP

$$
\delta \equiv \frac{m_{\mathrm{NLSP}}-m_{\mathrm{LSP}}}{m_{\mathrm{LSP}}}=\frac{\Delta M}{m_{\mathrm{LSP}}}
$$

In the degenerate gravitino scenario, the total dark matter abundance has two sources: one is

\footnotetext{
${ }^{2}$ In principle, in gauge mediation or anomaly mediation models, constraints from gravitino decays can be evaded as gravitino mass can be, respectively, much smaller or much larger than typical SUSY masses
} 
the usual LSP component from thermal production and decoupling and a second one from the NLSP non-thermal decays to LSP and SM particles. The sum of both components should reproduce the observed CDM relic density. The phenomenology of the degenerate gravitino scenario depends on both the identity of the lightest MSSM supersymmetric particle and on whether the gravitino is the LSP or the NLSP. The BBN, CMB and DEBRA bounds apply to the NLSP abundance while the LSP abundance is only constrained by the total dark matter abundance. In most cases, the lightest MSSM supersymmetric particle can be either the lightest neutralino or the lightest stau. Given that dark matter can not be a charged particle, we are left with three possibilities: gravitino LSP with neutralino or stau NLSP and gravitino NLSP with neutralino LSP. We will see that, for lifetimes smaller than the age of the universe, the strongest constraints on the reheating temperature arise in the case of gravitino NLSP, where all the previous constraints apply to the gravitino abundance and thus on the reheating temperature. On the other hand, when the gravitino is the LSP, it is possible to reach reheating temperatures compatible with thermal leptogenesis provided that the NLSP abundance at decoupling is sufficiently suppressed. Clearly, if the NLSP lifetime is much longer than the age of the universe, corresponding to small enough $\delta$, the maximal reheating temperatures consistent with the observed DM abundance $\left(T_{\mathrm{RH}} \simeq 4.1 \times 10^{9} \mathrm{GeV}\right)$ can be reached.

In this work, we will reanalyze the different constraints on the energy injected by NLSP decays and the reheating temperature in terms of this parameter $\delta$ in the three above mentioned cases. In the next section, we will present the constraints on the energy release for different lifetimes of the NLSP in a model independent way. In Section 3 we apply these constraints to the gravitino case where the energy release and the lifetime are related. In section 4 we analyse the case of the CMSSM looking for the new constraints on the reheating temperature in the degenerate gravitino scenario. Finally in section 5 we present our conclusions.

\section{Model-independent bounds}

If the NLSP lifetime is smaller than the age of the Universe, the energetic decay products can significantly affect cosmology. Even for lifetimes slightly larger than the age of the universe, observation of diffuse gamma rays can constraint this scenario. In this section, we consider the model-independent constraints on the degenerate NLSP-LSP scenario. As we will see, depending on the NLSP lifetime, these constraints originate from BBN observations, $\mathrm{CMB}$ spectral distortion or searches of diffuse gamma rays. There are additional constraints if the NLSP is charged. We focus on the case that the NLSP and LSP masses are nearly degenerate i.e. $\delta \ll 1$ in Eq. (10).

\subsection{Relic abundance constraint}

The first constraint that should be considered is that the total relic density of cold dark matter must match the observed value. We will consider the situation where only the LSP and the NLSP are relevant; that is, heavier MSSM particles have already decayed to LSP/NLSP before BBN takes place. In general, the final abundance of the LSP will have two components. The first one is 
the thermal abundance, $\Omega_{\mathrm{LSP}}^{\mathrm{TP}}$, which comes from thermal processes occurring in the plasma like scatterings and freeze-out. The second one is the non-thermal component, $\Omega_{\mathrm{LSP}}^{\mathrm{NTP}}$, that includes the contribution from LSP particles produced in NLSP decay. Therefore, the total cold dark matter relic density, will be 3

$$
\Omega_{\mathrm{CDM}} h^{2}=\Omega_{\mathrm{LSP}}^{\mathrm{TP}} h^{2}+\frac{1}{1+\delta} \Omega_{\mathrm{NLSP}}^{\mathrm{TP}} h^{2},
$$

where the last term stands for $\Omega_{\mathrm{LSP}}^{\mathrm{NTP}}$ and we have used Eq. (1). Assuming no late entropy release, Eq. (2) represents the present amount of dark matter, which should be equated with the observed value [5]

$$
\Omega_{\mathrm{WMAP}} h^{2}=0.1131 \pm 0.0034
$$

It is useful to define the new parameter

$$
\omega \equiv \frac{Y_{\mathrm{NLSP}}}{Y_{\mathrm{CDM}}}
$$

which quantifies the amount of present cold dark matter coming from the NLSP decay. In this equation $Y_{\mathrm{NLSP}}$ refers to the NLSP yield just before its decay 4. Combining Eq. (2) and Eq. (4) we get that

$$
\omega=1-\frac{\Omega_{\mathrm{LSP}}^{\mathrm{TP}} h^{2}}{\Omega_{\mathrm{WMAP}} h^{2}},
$$

which implies that $\omega \leq 1$ independently of $\delta$.

\subsection{BBN constraints}

At temperatures of order $T \sim 1 \mathrm{MeV}$, the light nuclei are synthesized in the primordial plasma. These temperatures corresponds to times between $1 \mathrm{sec}$ and $10^{3} \mathrm{sec}$. The obtained abundances in standard BBN calculations are in striking agreement with observation 5 . However, the injection of energetic particles in the primordial plasma at BBN or later can disrupt the standard BBN processes [8, 9, 10, 11, leading to a disagreement between theory and observation. Thus, any additional particle decaying at BBN or later is subject to the strong constraints from light nuclei abundances. Usually, the energy of injected particles is assumed to be of the same order of the LSP/NLSP mass and stringent bounds were derived for various scenarios from BBN [12, 13, 14, 15, 16, 17, 18, 19, 20, 21,. These constraints are obtained by solving the full set of Boltzmann equations for BBN with a latedecaying particle. This requires the detailed study of the spectrum of decay products with all the relevant nuclear cross sections [9, 10, 11]. The constraints apply to the released electromagnetic or

\footnotetext{
${ }^{3}$ Recall that we are assuming that the NLSP lifetime is smaller than the age of the Universe. If it were not the case, Eq. (2) is still valid as we are considering $m_{\mathrm{NLSP}} \simeq m_{\mathrm{LSP}}$.

${ }^{4}$ Here the yield of a species $i$ is defined as the ratio of the number density $n_{i}$ to entropy, $Y_{i} \equiv n_{i} / s$. Recall also that the yield and the relic density of a massive particle species $i$ are related through $Y_{i} \simeq$ $4.1 \times 10^{-12}\left(100 \mathrm{GeV} / m_{i}\right)\left(\Omega_{i} h^{2} / 0.11\right)$.

${ }^{5}$ There are possible discrepancies in Lithium abundances, which might be explained using Gravitino dark matter [6, [7].
} 
hadronic energy, parametrised by $\xi_{i}$ defined as 13 .

$$
\xi_{i} \equiv E_{i} B_{i} Y_{\mathrm{NLSP}}
$$

where $E_{i}$ is the released energy per decay with $i=$ em for electromagnetic decays and $i=$ had for the hadronic ones and $B_{i}$ stands for the respective branching ratios. The constraints from hadronic processes are important when the lifetime is relatively short $\tau_{\mathrm{NLSP}} \lesssim 10^{7} \mathrm{sec}$ [10, 16]. The typical lifetimes considered in this work are larger that $10^{7} \mathrm{sec}$ and therefore, in the following, we will consider only the BBN constraints on $\xi_{\mathrm{em}}$.

In the 2-body electromagnetic decay, the corresponding released energy is

$$
E_{\mathrm{em}}=\frac{m_{\mathrm{NLSP}}^{2}-m_{\mathrm{LSP}}^{2}}{2 m_{\mathrm{NLSP}}},
$$

where we assumed that the visible particle mass is negligible. In the degenerate mass limit, $\delta \ll 1$, this reduces to $E_{\mathrm{em}} \simeq m_{\mathrm{LSP}} \delta$. Notice that, as emphasized before, $E_{\mathrm{em}}$ is much smaller than the usually 6 assumed value $m_{\mathrm{NLSP}} / 2$.

Given the above discussion, for our purposes, we can apply the constraints on $\xi_{\mathrm{em}}$ from Ref. [1] directly to our scenario. Then, the constraint on $\xi_{\mathrm{em}}$ reads

$$
\xi_{\mathrm{em}} \simeq 4.1 \times 10^{-10} \mathrm{GeV}\left(\frac{\Omega_{\mathrm{WMAP}} h^{2}}{0.11}\right) \omega B_{\mathrm{em}} \delta<\xi_{\text {upper limit }},
$$

where the right hand side in the above equation can be read off from the upper limit derived in [11] for $B_{\text {had }}=0$.

For general values, the upper limit on the product $\omega B_{\mathrm{em}} \delta$ from BBN is shown in Figure 1 with red (dashed) line. From this figure, we can see that for lifetimes between $10^{7}$ and $10^{10}$ sec, this constraint requires $\omega B_{\mathrm{em}} \delta$ to be between $10^{-3}$ and $10^{-4}$. Notice that BBN constraints apply equally to the case of charged NLSP decaying to gravitino and electromagnetic showers.

\subsection{CMB spectral distortion}

In addition to $\mathrm{BBN}$ constrains, for long lifetimes $\tau_{\mathrm{NLSP}} \gtrsim 10^{7}$ sec., there are strong bounds from the shape of the CMB black-body spectrum. As pointed out in [12, the late injection of electromagnetic energy may distort the frequency dependence of the CMB spectrum from its observed blackbody shape. At late times of interest in our scenario, energetic photons from NLSP decays lose energy through processes such as $\gamma e^{-} \rightarrow \gamma e^{-}$, but photon number remains conserved since other processes, like double Compton scatterings and thermal bremsstrahlung, become inefficient. As a result, the spectrum follows the Bose-Einstein distribution function

$$
f_{\gamma}(E)=\frac{1}{e^{E /(k T)+\mu}-1},
$$

where $\mu$ here denotes the chemical potential. Then, the chemical potential of the distorted CMB spectrum has to satisfy the constraint, $|\mu|<9 \times 10^{-5}$ [23], which, for decay lifetimes $\tau_{\mathrm{NLSP}} \lesssim$

\footnotetext{
${ }^{6}$ Small mass differences has been considered in [21, 22, and for completely different motivations than ours.
} 


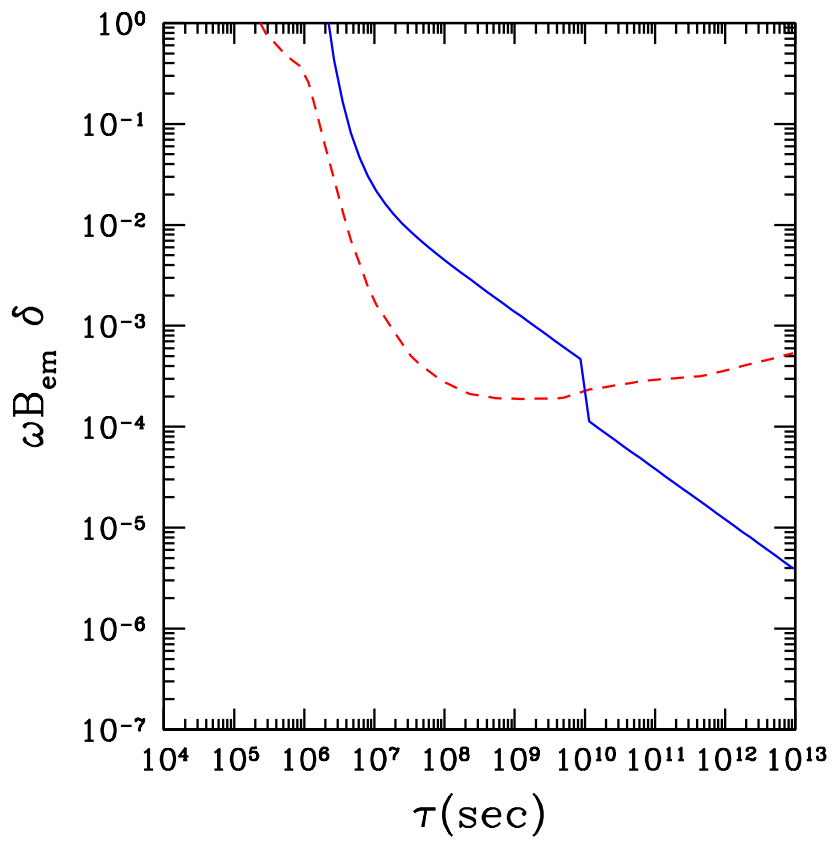

Figure 1: Constraints on the combined parameters $\omega B_{\mathrm{em}} \delta$ versus lifetime of NLSP using the result of [11] with $B_{h}=0$. The red (dashed) line come from em BBN constraints and blue (solid) line from CMB distortion as explained in the text. Regions above the lines are excluded.

$8.8 \times 10^{9} \mathrm{sec}$, translates into an upper bound on the released energy from NLSP decay, $\xi_{\text {em }}$ defined in Eq. (6) [24, 15],

$$
\xi_{\mathrm{em}}<1.59 \times 10^{-8} e^{\left(\tau_{d C} / \tau_{\mathrm{NLSP}}\right)^{5 / 4}}\left(\frac{1 \mathrm{sec}}{\tau_{\mathrm{NLSP}}}\right)^{1 / 2} \mathrm{GeV},
$$

where $\tau_{d C} \simeq 6.085 \times 10^{6} \mathrm{sec}$.

For longer lifetimes $\left(\tau_{\mathrm{NLSP}} \gtrsim 8.8 \times 10^{9} \mathrm{sec}\right)$, the spectral distortions in the CMB spectrum can be described in terms of the integral of the fractional contributions to the energy $\epsilon$ of the CMB per comoving volume during decay through the Compton $y$ parameter, $4 y=\delta \epsilon / \epsilon$, given by

$$
\frac{\delta \epsilon}{\epsilon}=7.04 \times \frac{1}{T\left(t_{\mathrm{eff}}\right)} \xi_{\mathrm{em}},
$$

where $T(t)$ is the CMB temperature and $t_{\mathrm{eff}}=[\Gamma(1-\beta)]^{1 / \beta} \tau_{\mathrm{NLSP}}$, for a time-temperature relation $T \propto t^{-\beta}$, with $\Gamma$ the usual Gamma function. In the radiation dominated era in the early Universe, for $T<0.1 \mathrm{MeV}$,

$$
T=1.15 \times 10^{-3}\left(\frac{t}{1 \mathrm{sec}}\right)^{-1 / 2} \mathrm{GeV}
$$

which gives $\beta=1 / 2$. Thus $t_{\text {eff }}=[\Gamma(1 / 2)]^{2} \tau_{\mathrm{NLSP}}=\pi \tau_{\mathrm{NLSP}}$.

The observational limit $|y|<1.2 \times 10^{-5}[25$ ] gives the constraint

$$
\xi_{\mathrm{em}} \lesssim 4.42 \times 10^{-9} \mathrm{GeV} \sqrt{\frac{1 \mathrm{sec}}{\tau_{\mathrm{NLSP}}}} .
$$


Using Eqs. (1113), this CMB constraint is plotted in Figure 1 with blue (solid) line. We can see that for lifetimes $\tau_{\mathrm{NLSP}} \gtrsim 10^{10} \mathrm{sec}$. the CMB constraint becomes more stringent than the BBN ones and sets the constraint on $\xi_{\mathrm{em}}$ until recombination time. Similarly to the BBN, CMB constraints apply also to charged NLSP decaying to gravitino and electromagnetic showers.

\subsection{Diffuse Gamma-ray observation}

After recombination, at the cosmic time around $10^{13} \mathrm{sec}$, the number density of free electrons drops quickly and the photons are almost free from the interactions. Therefore the photons from the decaying particles can reach us now and contribute to the Cosmic Gamma-ray Background (CGB). The observed CGB highly constrains any extra contribution including the photons from late decaying particles 7 .

The present photon flux from two-body decay can be written as

$$
\frac{d \Phi}{d E_{\gamma}}=\frac{c}{4 \pi} \int_{t_{i}}^{t_{0}} \frac{d t}{\tau_{\mathrm{NLSP}}} \frac{\rho_{c} \Omega_{\mathrm{WMAP}} \omega B_{\mathrm{em}}}{m_{\mathrm{NLSP}}} e^{-t / \tau_{\mathrm{NLSP}}} \delta\left(E_{\gamma}-a E_{\mathrm{em}}\right)
$$

where $E_{\mathrm{em}} \simeq m_{\mathrm{LSP}} \delta$ is the energy of the photon at production, $\tau_{\mathrm{NLSP}}$ and $m_{\mathrm{NLSP}}$ are the lifetime and mass of NLSP, $\rho_{c}=3 H_{0}^{2} / 8 \pi G_{N}=8.0992 h^{2} \times 10^{-47} \mathrm{GeV}^{4}$ and $a=a(t)$ is the time-dependent scale factor with $a\left(t_{0}\right)=1$ at present time $t_{0}$. The delta function can be integrated using the formula $\delta(f(t))=\delta\left(t-t_{a}\right) /\left|f^{\prime}\left(t=t_{a}\right)\right|$ where $t_{a}$ is the solution which satisfies $E_{\gamma}=a\left(t_{a}\right) E_{\mathrm{em}}$. After integration, the flux reads

$$
\frac{d \Phi}{d E_{\gamma}}=\frac{c}{4 \pi} \frac{\rho_{c} \Omega_{\mathrm{WMAP}} \omega B_{\mathrm{em}}}{m_{\mathrm{NLSP}} \tau_{\mathrm{NLSP}}} \frac{e^{-t_{a} / \tau_{\mathrm{NLSP}}}}{E_{\gamma} H\left(E_{\gamma} / E_{\mathrm{em}}\right)} \Theta\left(E_{\mathrm{em}}-E_{\gamma}\right)
$$

where the $\Theta$ function simply cuts energies larger than the initial energy and each observed photon with energy $E_{\gamma}$ is produced at a time $t_{a}$ which satisfies $a\left(t_{a}\right)=E_{\gamma} / E_{\mathrm{em}}$. Assuming the dark energy is a cosmological constant, this function $t_{a}(a)$ is given by [22]

$$
t_{a} \equiv t\left(a=E_{\gamma} / E_{\mathrm{em}}\right)=\frac{2 \log \left[\left(\sqrt{\Omega_{\Lambda} a^{3}}+\sqrt{\Omega_{M}+\Omega_{\Lambda} a^{3}}\right) / \sqrt{\Omega_{M}}\right]}{3 H_{0} \sqrt{\Omega_{\Lambda}}},
$$

where $H(a)=H_{0} \sqrt{\Omega_{M} a^{-3}+\Omega_{\Lambda}}$.

Taking into account that $c / H_{0}=1.3 \times 10^{28} \mathrm{~cm}, \rho_{c}=5.6 \times 10^{-6} \mathrm{GeV} / \mathrm{cm}^{3}, H_{0}=70 \mathrm{~km} \mathrm{sec}^{-1} \mathrm{Mpc}^{-1}$, $\Omega_{\Lambda}=0.7, \Omega_{M}=\Omega_{\mathrm{CDM}}+\Omega_{B}=0.3$ and $\Omega_{\mathrm{CDM}}=\Omega_{\mathrm{WMAP}}=0.25$, we find that the flux is

$$
\frac{d \Phi}{d E_{\gamma}}=1.37 \times 10^{21} \mathrm{GeV} \mathrm{cm}^{-2} \frac{\omega B_{\mathrm{em}}}{m_{\mathrm{NLSP}} \tau_{\mathrm{NLSP}}} \frac{e^{-t_{a} / \tau_{\mathrm{NLSP}}}}{E_{\gamma} \sqrt{0.7+0.3\left(E_{\mathrm{em}} / E_{\gamma}\right)^{3}}},
$$

with

$$
t_{a}=3.51 \times 10^{17} \sec \log \left[\sqrt{2.33 a^{3}}+\sqrt{1+2.33 a^{3}}\right] .
$$

\footnotetext{
${ }^{7}$ Under appropriate conditions, the late decays of WIMPs to gravitinos and $\mathrm{MeV}$ photons may explain the MeV CGB anomalies [22].
} 
This differential flux must be compared to the observation of diffuse gamma ray flux. At each photon energy, we must require that this flux is smaller than the flux, $E_{\gamma}^{2} \frac{d \Phi}{d E_{\gamma}}$, observed by SPI, COMPTEL and EGRET [3]. Notice that, unlike the BBN and CMB constraints where we can find a bound on $\xi_{\mathrm{em}}$ for a given value of $\tau_{\mathrm{NLSP}}$, now we need to specify both $\tau_{\mathrm{NLSP}}$ and $E_{\gamma}$ to obtain a bound on $\omega B_{\mathrm{em}} \delta$.

On the other hand, if the lifetime of NLSP is longer than the age of Universe, the line spectrum from the galactic center can be observed without cosmological redshift and this provides a further constraint on the emitted radiation from NLSP decays. For this we apply the bounds from Ref. [3]. Taking into account of the exponential decay of NLSP we have

$$
\frac{\rho_{s c} \omega B_{\mathrm{em}}}{4 \pi m_{\mathrm{NLSP}} \tau_{\mathrm{NLSP}}} e^{-t_{0} / \tau_{\mathrm{NLSP}}} \zeta_{\text {lim }}<\mathcal{F}\left(E_{\gamma}=E_{\mathrm{em}}\right),
$$

where $\rho_{s c}=0.3 \mathrm{GeV} \mathrm{cm}^{-3}$ is the dark matter density at the solar distance from the Galactic center, $R_{s c}=8.5 \mathrm{kpc}, \zeta_{\text {lim }}$ is a dimensionless integral of the line-of-sight intensity in the galactic center which ranges between $0.5-1.5$ for various dark matter halo profiles [3]. The function $\mathcal{F}$ is given in Figure 2 of Ref. 3]. Once again, as in the case of the CGB constraint in Eq. (17), this constraint depends on the photon energy and therefore we need to specify both $\tau_{\mathrm{NLSP}}$ and $E_{\gamma}$ to obtain bound on $\omega B_{\mathrm{em}} \delta$. Thus, there is no simple analog of Figure 1 for a constraint on $\xi_{\mathrm{em}}$ from Diffuse gamma rays observations. Moreover, the constraints on $\xi_{\mathrm{em}}$ from the galactic center gamma rays are of the same order of magnitude (although slightly stronger) as the ones from the diffuse extragalactic emission. Therefore, for simplicity, we will only consider the diffuse gamma rays constraints, which apply to a broader range of energy.

\subsection{Catalyzed BBN}

Heavy long-lived negatively charged particles, $X^{-}$, present during BBN can bind with light nuclei modifying standard BBN reactions. These catalyzed reactions, called CBBN, result in a change of light element abundances, and in particular lead to the overproduction of ${ }^{6} \mathrm{Li}$ through $\mathrm{CBBN}$ reactions [26]. For lifetimes longer than $5 \times 10^{3} \mathrm{sec}$, the observed light-nuclei abundances result on a constraint on the abundance of the charged relic, $Y_{X^{-}}<2 \times 10^{-16}$ [27, 28]. However, taking more conservative ${ }^{6} \mathrm{Li} /{ }^{7} \mathrm{Li}$ constraints, it is possible to relax slightly the previous bound to $Y_{X^{-}}<10^{-14_{-}}$ $10^{-15}[29,30]$.

Using Eq. (44), we can translate the constraint on the yield of charged NLSP from catalyzed BBN to a bound on $\omega$

$$
\omega \lesssim 2.44 \times 10^{-3}\left(\frac{m_{\mathrm{LSP}}}{100 \mathrm{GeV}}\right)\left(\frac{Y_{\mathrm{CBBN}}}{10^{-14}}\right)
$$

where we used $\Omega_{\mathrm{WMAP}} h^{2}=0.11$ and $Y_{\mathrm{CBBN}}$ is the maximum value allowed from catalyzed BBN for lifetimes larger than $10^{5} \mathrm{sec}$. As can be see from this equation, the catalyzed BBN bound is very stringent and indeed, it is very difficult to obtain such small yield at freeze-out in the MSSM [31]. However, it is still possible to find small allowed regions in the MSSM, or even in the CMSSM, where the $\widetilde{\tau}$ is the NLSP with large $\tan \beta$ [32, 33] with relaxed ${ }^{6} \mathrm{Li} /{ }^{7} \mathrm{Li}$ bounds [21]. 


\section{Bounds on the degenerate gravitino scenario}

In this section, we will apply the model-independent bounds derived in the last section to the case of degenerate gravitino scenario. In our scenario, the gravitino can be either the LSP or the NLSP. In both cases it is thermally produced at reheating. After inflation, the Universe is reheated at a temperature $T_{\mathrm{RH}}$ and gravitinos are produced through thermal scatterings in the plasma 8 . Their resulting relic density is linear in the reheating temperature and it is given by [35]

$$
\Omega_{3 / 2}^{\mathrm{TP}} h^{2} \simeq 0.27\left(\frac{T_{\mathrm{RH}}}{10^{10} \mathrm{GeV}}\right)\left(\frac{100 \mathrm{GeV}}{m_{3 / 2}}\right)\left(\frac{M_{3}}{\mathrm{TeV}}\right)^{2},
$$

where $M_{3}$ is the gluinos mass. Assuming no late entropy release, Eq. (44) sets an absolute bound on the reheating temperature, i.e. $\Omega_{3 / 2}^{\mathrm{TP}} \lesssim \Omega_{\mathrm{WMAP}}$, implies

$$
T_{\mathrm{RH}} \lesssim 4.1 \times 10^{9} \mathrm{GeV}\left(\frac{m_{3 / 2}}{100 \mathrm{GeV}}\right)\left(\frac{\mathrm{TeV}}{M_{3}}\right)^{2}
$$

This has to be compared with the minimum reheating temperature for successful thermal leptogenesis [37, 38, 39] $T_{\mathrm{RH}} \gtrsim 2 \times 10^{9} \mathrm{GeV}$. From this equation it is clear that the reheating temperature can not reach values much above $\sim 10^{10} \mathrm{GeV}$. 10 In addition to this constraint, we have to implement the constraints on the released energy considered in the last section.

The relevant particles in the analysis of NLSP decays in the degenerate scenario are the gravitino and the lightest MSSM particle, which can be either the neutralino or the stau11. We have two different situations depending on the particle nearly degenerate with the gravitino, namely gravitinoneutralino and gravitino-stau degeneracy. In each case the gravitino can be either the LSP or the NLSP.

\subsection{Gravitino-neutralino degeneracy}

As usual, in the MSSM, the lightest neutralino eigenstate $\chi_{1}^{0}$ is parametrised as $\chi_{i}^{0}=N_{i 1}(-i \widetilde{B})+$ $N_{i 2}\left(-i \widetilde{W}_{3}\right)+N_{i 3} \widetilde{H}_{U}^{0}+N_{i 4} \widetilde{H}_{D}^{0}$, where the unitary matrix $N$ defines the composition of neutralinos in terms of the Bino, Wino and Higgsinos. Since we are considering mass splittings that are smaller than the $Z$ mass to suppress the hadronic branching ratio, the dominant (2-body) decay channel will be $\chi_{1}^{0} \rightarrow \gamma \widetilde{G}$ or $\widetilde{G} \rightarrow \chi_{1}^{0} \gamma$. As we will see below, the typical lifetime of NLSP is of order $10^{13} \mathrm{sec} \times(1 \mathrm{GeV} / \Delta M)^{3}$. Therefore, depending on $\Delta M$ we will have to consider different constraints.

\footnotetext{
${ }^{8} \mathrm{We}$ assume that at $T_{\mathrm{RH}}$, the Universe is composed of a thermal bath of MSSM degrees of freedom and that gravitinos production by inflaton decay is negligible 34 .

${ }^{9}$ Taking into account the result of Ref. $[36$ the gravitino relic density would roughly increase a factor 2 .

${ }^{10} \mathrm{~A}$ possibility to increase $T_{\mathrm{RH}}$ is to consider a squeezed gaugino spectrum reducing the gluino mass $M_{3}$ (see e.g. [40]).

${ }^{11}$ Sneutrinos LSP are marginally allowed [41] due to their too large direct detection cross-sections. We do not consider them in our analysis.
} 


\subsubsection{Gravitino LSP}

In this case, the lifetime of neutralinos is given by

$$
\tau_{\chi} \simeq \frac{1.78 \times 10^{13} \mathrm{sec}}{\left|N_{11} \cos \theta_{W}+N_{12} \sin \theta_{W}\right|^{2}}\left(\frac{1 \mathrm{GeV}}{\Delta M}\right)^{3} .
$$

Notice that, in the limit of $\Delta M \ll m_{3 / 2}$, the lifetime depends only on the mass splitting $\delta \times$ $m_{3 / 2}=\Delta M$, but not on the overall mass scale $m_{3 / 2}$ [4, 13. If the gravitino is the LSP, the neutralino will decay into gravitino and photon and there are strong constraints on the released energy $\xi_{\mathrm{em}}=\omega B_{\mathrm{em}} \delta$. Different constraints will apply depending on the lifetime which, in turn is fixed by $\Delta M$.

- For $10 \mathrm{GeV} \lesssim \Delta M \lesssim 90 \mathrm{GeV}$, the relevant constraint is BBN and from Fig. 1 we have roughly $\omega \delta \lesssim 10^{-3}$.

- For smaller splittings, from $1 \mathrm{GeV} \lesssim \Delta M \lesssim 10 \mathrm{GeV}$, the constraints from CMB spectrum are much stronger than the BBN ones and $\omega \delta$ is between $10^{-4}$ and $10^{-6}$

- For $30 \mathrm{MeV} \lesssim \Delta M \lesssim 1 \mathrm{GeV}$ we have to take into account the diffuse gamma ray observations. Although the constrains on $\omega \delta$ depend on $m_{3 / 2}$, for $m_{3 / 2} \simeq 100 \mathrm{GeV}$, typical values range from $\omega \delta=10^{-6}$ to $\omega \delta=10^{-9}$.

- Mass differences from $2 \mathrm{MeV} \lesssim \Delta M \lesssim 30 \mathrm{MeV}$ correspond to NLSP with lifetimes $2 \times$ $10^{21} \mathrm{sec} \gtrsim \tau_{\mathrm{NLSP}} \gtrsim 5 \times 10^{17} \mathrm{sec}$. These NLSP are already decaying at present, therefore diffuse gamma ray observations constrain their abundance. In this case $\omega \delta$ ranges from $10^{-9}$ to $10^{-5}$.

- Finally, for smaller $\Delta M$, the neutralino is still present in the universe and it is completely stable for practical purposes. The only constraint comes from the WMAP measurement of the dark matter abundance.

These constraints are summarized in Figure 2, We can see that for a given $\Delta M$ the constraints on $\omega$ are very strong for $\Delta M>30 \mathrm{MeV}$, and, as we will see, it is difficult to reach such small neutralino abundances at decoupling in the MSSM. For smaller $\Delta M<2 \mathrm{MeV}$, corresponding to neutralino lifetimes longer than the age of the universe, $\omega \simeq 1$ is evidently allowed. For $2 \mathrm{MeV}<\Delta M<30 \mathrm{MeV}$, $\omega$ ranges from 1 to $10^{-6}$.

The only constraint on the gravitino abundance comes from Eq. (2) and the dark matter abundance measured by WMAP. Given $\omega$, we can use Eq. (11) and Eq. (4) to calculate the required reheating temperature

$$
T_{\mathrm{RH}}=4.1 \times 10^{9} \mathrm{GeV}\left(\frac{m_{3 / 2}}{100 \mathrm{GeV}}\right)\left(\frac{1 \mathrm{TeV}}{M_{3}}\right)^{2}(1-\omega) .
$$

From Eq. (24), we see that, provided $\omega \ll 1$, one gets the maximal allowed reheating temperature $T_{\mathrm{RH}}=4.1 \times 10^{9} \mathrm{GeV}$. 


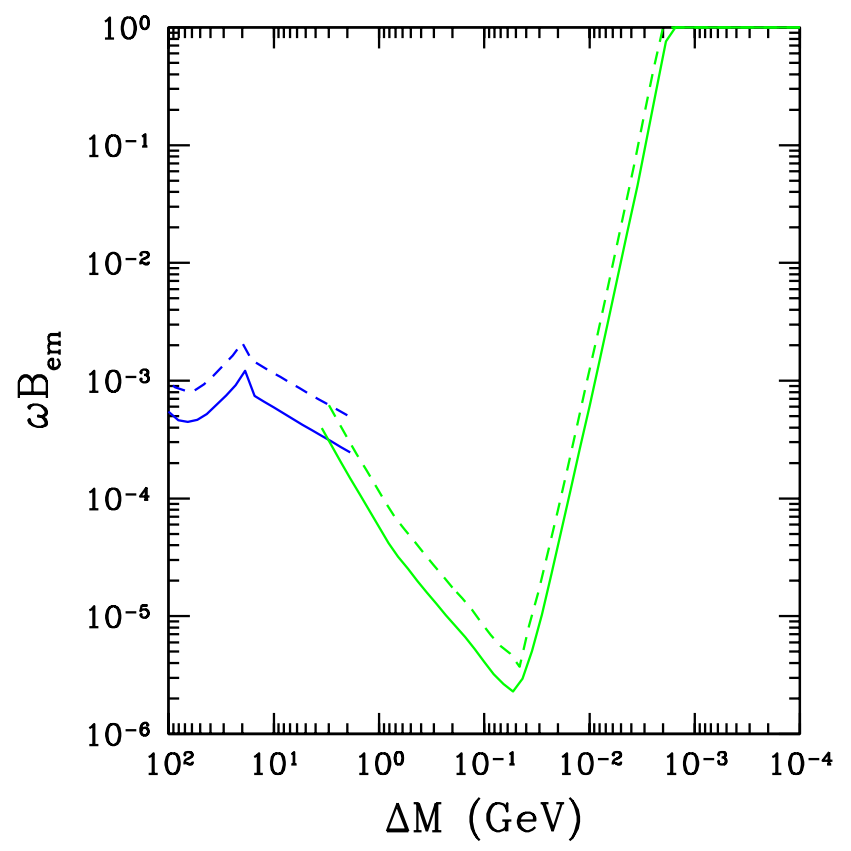

Figure 2: Constraints on the combined parameters $\omega B_{\text {em }}$ versus $\Delta M$ for given mass of neutralino NLSP (for $N_{11} \simeq 1$ ) with Gravitino LSP. Solid line is for $m_{\mathrm{NLSP}}=100 \mathrm{GeV}$ and dashed line for $m_{\mathrm{NLSP}}=200 \mathrm{GeV}$. The blue lines for $\Delta M>2 \mathrm{GeV}$ come from BBN and CMB constraints and the green lines for $\Delta M<2 \mathrm{GeV}$ come from the diffuse gamma ray observations. Regions above the lines are excluded.

\subsubsection{Gravitino NLSP}

If the gravitino is the NLSP, the dominant decay channel is $\widetilde{G} \rightarrow \gamma \chi_{1}^{0}$ and its lifetime is given by

$$
\tau_{3 / 2} \simeq \frac{3.56 \times 10^{13} \mathrm{sec}}{\left|N_{11} \cos \theta_{W}+N_{12} \sin \theta_{W}\right|^{2}}\left(\frac{1 \mathrm{GeV}}{\Delta M}\right)^{3}
$$

which is only a factor 2 larger than the gravitino LSP case. However, unlike the gravitino LSP case, the bounds on the released energy from this decay constrain strongly the initial thermal abundance of gravitinos. Notice that, here, $\omega$ represents the fraction of neutralinos coming from gravitino decay and therefore the initial abundance of gravitinos.

The constraints on $\omega \delta$ for different ranges of $\Delta M$ seen in the previous section apply equally in this case. Now, given $\Delta M$ and $m_{3 / 2}$, which fix $\delta$, we have a direct constraint on the gravitino thermal abundance, and hence on $\omega$. As we know, the gravitino thermal abundance is directly proportional to $T_{\mathrm{RH}}$ which in this case can be written

$$
T_{\mathrm{RH}} \simeq 4.1 \times 10^{9} \mathrm{GeV}\left(\frac{m_{3 / 2}}{100 \mathrm{GeV}}\right)\left(\frac{1 \mathrm{TeV}}{M_{3}}\right)^{2} \omega\left(\frac{1}{1+\delta}\right) .
$$

From this equation, we see that, opposite to the case of gravitino LSP, in order to maximize the reheating temperature, one needs $\omega$ as large as possible. The constraints on $T_{\mathrm{RH}}$ as a function of $\delta$ 


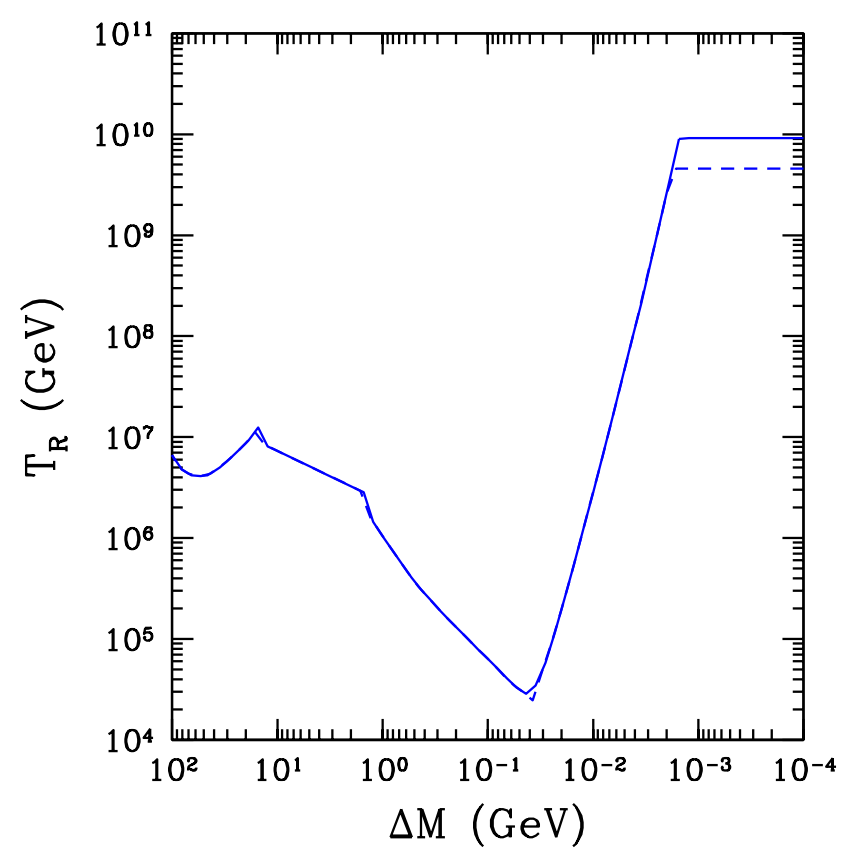

Figure 3: Constraints on the maximal reheating temperature after inflation for the Gravitino NLSP with neutralino LSP (with $N_{11} \simeq 1$ ) for $m_{\mathrm{NLSP}}=100 \mathrm{GeV}$ (solid) and $200 \mathrm{GeV}$ (dashed). Regions above the lines are excluded. Notice that, as we can see from Eq. (26), these maximal reheating temperatures are only possible if the corresponding bounds on $\omega$ are satisfied

are shown in Figure 3. Given the strong constraints on $\omega$ for the different $\delta \mathrm{s}, T_{\mathrm{RH}}$ is considerably smaller than the maximal value allowed by WMAP. The only exception to this are the cases with very small $\Delta M \lesssim 2 \times 10^{-3} \mathrm{GeV}$, corresponding to the NLSP still present as a dark matter component, where $\omega \lesssim 1$ and the LSP abundance is smaller that the observed $\Omega_{\mathrm{WMAP}} h^{2}$.

\subsection{Gravitino-stau degeneracy}

In the MSSM, the lightest stau state $\widetilde{\tau}_{1}$ can also be the LSP. However this situation is usually discarded as staus cannot play the role of cold dark matter. The remaining possibility is then that $\widetilde{\tau}_{1}$ is the NLSP with the gravitino as the LSP. The dominant decay channel in this case is $\widetilde{\tau}_{1} \rightarrow \tau \widetilde{G}$ if the mass-difference is larger than the tau mass, i.e. $\Delta M \geq 1.77 \mathrm{GeV} 12$. In this case, the lifetime

\footnotetext{
${ }^{12}$ Notice that for $\Delta M \leq 1.77 \mathrm{GeV}$ the two body flavour-conserving channel is closed and the stau can decay only through lepton flavour violating channels, $\widetilde{\tau}_{1} \rightarrow \mu \widetilde{G}$ or $\widetilde{\tau}_{1} \rightarrow e \widetilde{G}$, where the lifetime would be inversely proportional to the lepton-flavour violating coupling [42, 43]: $\tau_{\widetilde{\tau}_{1}} \simeq 2 \times 10^{14} \mathrm{sec}\left|\delta_{\tau i}^{\mathrm{LFV}}\right|^{-2}$ for $m_{3 / 2}=100 \mathrm{GeV}$ and $\Delta M=2 \mathrm{GeV}$. This means that, if these flavour violating couplings, the so-called Mass Insertions, are sizable, $\delta_{\tau i}^{\mathrm{LFV}} \geq 0.03$, the stau could decay before the present time. However, we do not consider this possibility in this paper.
} 
of the stau NLSP is then given by

$$
\tau_{\widetilde{\tau}_{1}} \simeq 6.69 \times 10^{15} \sec \left(\frac{m_{3 / 2}}{300 \mathrm{GeV}}\right)\left(\frac{2 \mathrm{GeV}}{\Delta M}\right)^{4}\left(\frac{0.21}{1-m_{\tau}^{2} /(\Delta M)^{2}}\right)^{3 / 2} .
$$

Notice that now, the stau lifetime, in contrast with the degenerate gravitino-neutralino case, does depend on the overall mass scale $m_{3 / 2}$. Moreover for a mass difference similar to the tau mass, $\Delta M \simeq 1.78 \mathrm{GeV}$, the stau lifetime is equal to the age of the universe.

In principle, the stau has to satisfy similar constraints as in the degenerate gravitino-neutralino case with the exception of diffuse gamma ray constraints. Notice that the stau does not decay directly to photons and therefore does not contribute to the gamma ray background. Despite this fact, all the decays of the stau produce electromagnetic cascades that affect both BBN and CMB observables.

Nevertheless, the main difference with the neutralino case is that stau, being charged, can form bound states with light elements and affect BBN predictions [28, 29]. Then, from Eq. (20) we obtain a strong constraint on $\omega$ which for $Y_{\mathrm{CBBN}}=10^{-15}$ is $\omega<7.32 \times 10^{-4} m_{\tilde{\tau}} /(300 \mathrm{GeV})$.

Finally, if the stau lifetime is longer than the age of the universe, the stau yield is very strongly bounded by the presence of anomalously heavy Hydrogen in deep sea water [44]. In terms of $\omega$ the bound reads, $\omega \leq 2.2 \times 10^{-27}\left(m_{\tilde{\tau}} / 100 \mathrm{GeV}\right)$, for stau masses between $5 \mathrm{GeV}$ and $1.7 \mathrm{TeV}$. Thus, in practice, this possibility can be completely discarded. In our analysis, we require that staus have already decayed at present and therefore we eliminate the staus with a lifetime longer than the age of the universe which corresponds to $\Delta M \lesssim 2 \mathrm{GeV}$.

These constraints are presented in Figure 4 where we see that for $Y_{\mathrm{CBBN}}=10^{-15}$ the CBBN is the most stringent of all the constraints and requires $\omega \lesssim 7 \times 10^{-4}$. This corresponds to stau relic density well below the observed relic density, Eq. (3), and the thermal relic density at freeze-out for purely RH staus [31]. In order to get such small abundance, the staus must have a substantial LR mixing. This requires both large $\mu$ and large $\tan \beta$ and moderate $m_{\widetilde{\tau}_{1}}$ [32, 33], which as we will see in section 4, is difficult, but still possible in the CMSSM. Furthermore, the vertical line at $\Delta M \simeq 2 \mathrm{GeV}$ corresponds to our requirement that all staus have already decayed at the present age.

The bound on $T_{\mathrm{RH}}$ here is analogous to the case of gravitino-neutralino degeneracy with gravitino LSP. Again, the reheating temperature is given in Eq. (24) and given that, in this case, the allowed points in parameter space require $\omega \lesssim 10^{-2}$, one gets the maximal reheating temperature $T_{\mathrm{RH}}=$ $4.1 \times 10^{9} \mathrm{GeV}$.

\section{The degenerate gravitino scenario in the CMSSM}

Perhaps the most appealing mechanism to transmit SUSY breaking from a hidden sector, where SUSY breaking occurs, to the visible sector is to use gravitational interactions, that are suppressed by the Planck scale 13 [45. This scenario is commonly called gravity mediation, and the CMSSM

\footnotetext{
${ }^{13}$ Of course, in gravity mediated scenarios, there can be an associated cosmological moduli problem. In this work, as this issue is outside the focus of the paper, we do not address it and we will just assume that it is solved by some unspecified mechanism.
} 


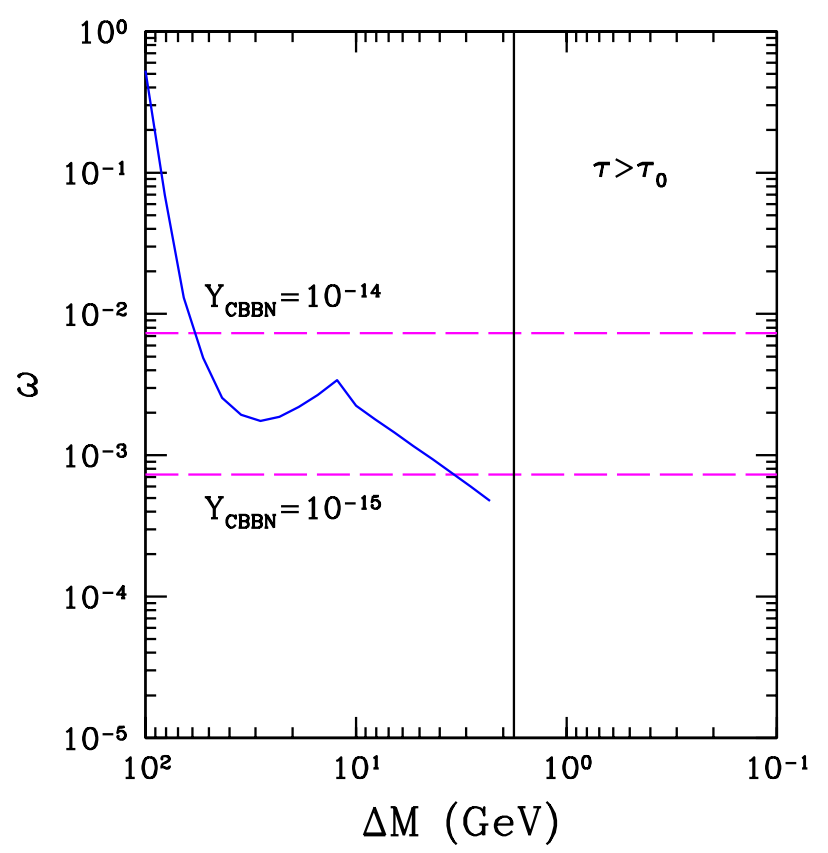

Figure 4: Constraints on $\omega$ versus $\Delta M$ with $m_{3 / 2}=300 \mathrm{GeV}$ for stau NLSP with Gravitino LSP. The solid blue line represents the BBN and CMB constraints. The vertical line at $\Delta M \simeq 2 \mathrm{GeV}$ corresponds to a stau lifetime equal to the age of the universe and therefore the region on the right is excluded from heavy-water searches. The dashed pink lines correspond to the CBBN constraint for $Y_{\mathrm{CBBN}}=10^{-14}$ and $Y_{\mathrm{CBBN}}=10^{-15}$.

is one of its simplest and most popular realizations for phenomenological studies [46]. It is defined in terms of only five free parameters: common scalar $\left(m_{0}\right)$, gaugino $\left(m_{1 / 2}\right)$ and tri-linear $\left(A_{0}\right)$ mass parameters (all specified at the GUT scale) plus the ratio of Higgs vacuum expectation values $\tan \beta$ and $\operatorname{sign}(\mu)$, where $\mu$ is the Higgs/higgsino mass parameter whose square is computed from the conditions of radiative electroweak symmetry breaking. In addition to these parameters, in this analysis we have the gravitino mass, $m_{3 / 2}$, that we keep as a free parameter.

The gravitino LSP (or NLSP) scenario in the CMSSM has been investigated thoroughly in the literature for the past years, both from the point of view of cosmological implications for dark matter [15] and for implications in collider searches [47]. One of the remarkable results of this scenario from cosmology is that the limits imposed on the reheating temperature of the Universe after an inflationary epoch have got down to a few $10^{7} \mathrm{GeVs}$ [21]. This constraint basically rules out the thermal leptogenesis mechanism as the mean to produce the observed baryon asymmetry of the universe.

In this section, we apply the results of the previous sections for the degenerate gravitino scenario to investigate the highest reheating temperature that can be reached in the CMSSM fulfilling the WMAP constraint on the CDM abundance and the relevant collider bounds; namely, direct SUSY 


\begin{tabular}{|l|lll|l|}
\hline Observable & \multicolumn{3}{|l|}{ Mean value } & \multicolumn{2}{|c|}{ Uncertainties } & ref. \\
& $\mu$ & $\sigma$ (exper.) & $\tau$ (theor.) & \\
\hline$\delta a_{\mu} \times 10^{10}\left(e^{+} e^{-}\right)$ & 29.5 & 8.8 & 2.0 & {$[48]$} \\
$B R\left(\bar{B} \rightarrow X_{s} \gamma\right) \times 10^{4}$ & 3.52 & 0.33 & 0.3 & {$[49]$} \\
$\Omega_{\chi} h^{2}$ & 0.1099 & 0.0062 & $0.1 \Omega_{\chi} h^{2}$ & {$[50]$} \\
\hline \hline & \multicolumn{2}{|l|}{ Limit $(95 \% \mathrm{CL})$} & $\tau$ (theor.) & ref. \\
\hline$m_{h}$ & $>114.4 \mathrm{GeV}$ & $3 \mathrm{GeV}$ & {$[51]$} \\
Sparticle masses & \multicolumn{2}{|c|}{ As implemented in Micromegas } & {$[52]$} \\
\hline
\end{tabular}

Table 1: Summary of the observables used in the analysis to constrain the CMSSM parameter space. Upper part: Observables for which a positive measurement has been made. $\delta a_{\mu}=a_{\mu}^{\exp }-a_{\mu}^{\mathrm{SM}}$ denotes the discrepancy between the experimental value and the SM prediction of the anomalous magnetic moment of the muon $(g-2)_{\mu}$. Lower part: Observables for which only limits currently exist.

searches, $(g-2)_{\mu}$ using $\left(e^{+} e^{-} \rightarrow\right.$ hadrons $)$ dat 14 and the $B R\left(B \rightarrow X_{s} \gamma\right)$. All the constraints imposed are summarized in Table 1 .

We use the fortran package SUSPECT [53] to solve the RGEs and to calculate the spectrum of physical sparticles and Higgs bosons, following the procedure outlined in [54]. To evaluate the CDM abundance in each point of the CMSSM parameter space, we employ the MicrOMEGAs code 52. The branching ratio for the $B \rightarrow X_{s} \gamma$ decay has been computed with the numerical code SusyBSG [55] using the full NLO QCD contributions, including the two-loop calculation of the gluino contributions presented in [56] and the results of [57] for the remaining non-QCD $\tan \beta$-enhanced contributions. Finally we compute $\delta_{\text {had }}^{\mathrm{SM}} a_{\mu}$ at full one-loop level adding the logarithmic piece of the quantum electrodynamics two-loop calculation plus two-loop contributions from both stop-Higgs and chargino-stop/sbottom [58]. Then, the effective two-loop effect due to a shift in the muon Yukawa coupling proportional to $\tan ^{2} \beta$ has been added as well [59]. Recall that the communication among the different codes is done via the SLHA accord 60.

In particular, regarding the direct constraints on new particle searches, given that the theoretical error in computing the lightest Higgs mass $m_{h}$ by SUSPECT is about $3 \mathrm{GeV}$ [61, we require the calculated value of $m_{h}$ to exceed $111 \mathrm{GeV}$. In the case of observables for which a positive measurement has been made, we require our predictions to be within the $2 \sigma$ range, for which we have added the theoretical and experimental errors, found in Table 1, in quadrature. In our numerical analysis we take $m_{t}=173.1 \mathrm{GeV}$ [62]. For the case of the stau NLSP, in addition, we completely exclude points that do not satisfy the conservative bound on $Y_{\widetilde{\tau}}<10^{-14}$ [29] from the catalyzed nuclear reactions [26] (see Fig. 4).

We are now ready to present some representative numerical results. We are going to calculate the abundance of the MLSP (MSSM LSP), $\mathrm{w}=Y_{\mathrm{MLSP}} / Y_{\mathrm{CDM}}$, at freeze-out. Notice that $\mathrm{w}=\omega$ if

\footnotetext{
${ }^{14}$ Notice that we obtain the hadronic contribution to $(g-2)_{\mu}$ using only the data from $\left(e^{+} e^{-} \rightarrow\right.$ hadrons), inclusion of the $\tau$ data can decrease the discrepancy between the SM prediction and the experimental result.
} 


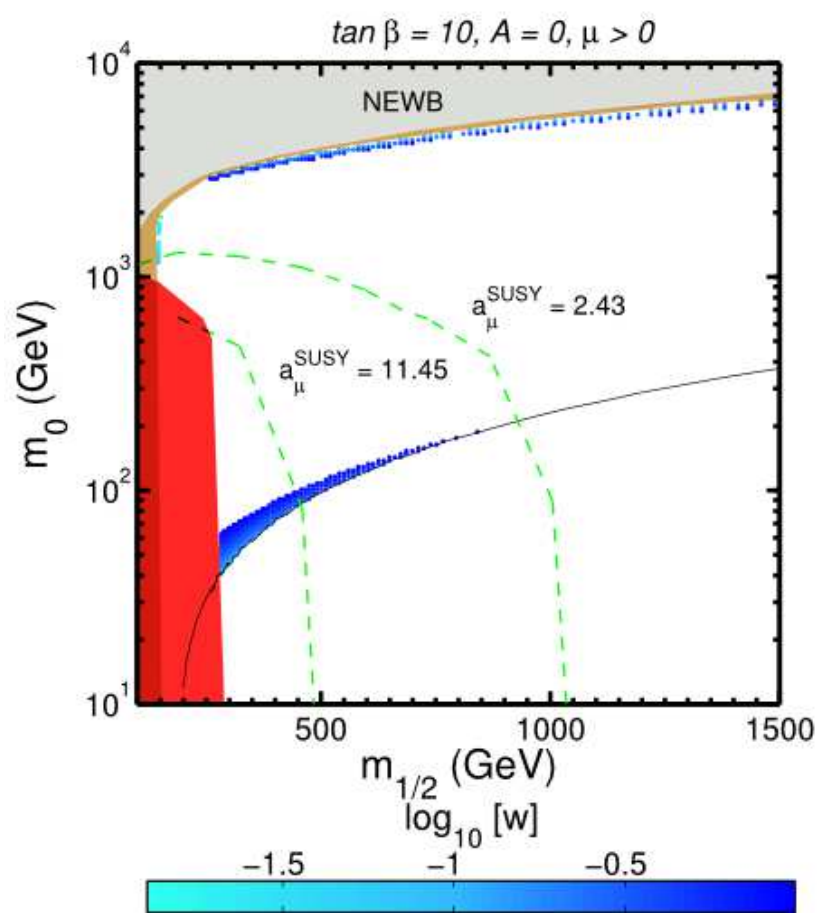

Figure 5: Values of $\mathrm{w}$ in the $m_{1 / 2}-m_{0}$ plane of the CMSSM parameter space for $m_{t}=173.1 \mathrm{GeV}$, $A_{0}=0, \tan \beta=10$ and $\mu>0$. The red (medium grey) region is forbidden by the Higgs bound from LEP and in the very light grey region no correct electroweak symmetry breaking is obtained. The light brown (light grey) band below the NEWB area corresponds to the region forbidden by the LEP chargino bound. The regions below the dashed green lines satisfy the $(g-2)_{\mu}$ constraint at the $2 \sigma\left(a_{\mu}^{\mathrm{SUSY}}>2.43 \times 10^{-10}\right)$ or $3 \sigma$ level $\left(a_{\mu}^{\mathrm{SUSY}}>11.45 \times 10^{-10}\right)$.

the MLSP is the NLSP, i.e. with gravitino LSP, but $\mathrm{w}=(1-\omega)$ when the gravitino is the NLSP. As a result, the reheating temperature will be proportional to $(1-\mathrm{w})$ in both cases. In the following we will focus on three representative $\tan \beta$ values: one moderate, $\tan \beta=10$, and two large values, $\tan \beta=50$ and $\tan \beta=55$, and we take $A_{0}=0$. Indeed, we have performed several other scans varying both $A_{0}$ and $\tan \beta$ and we found that there are no significant differences to these cases.

\subsection{Low-medium $\tan \beta=10$}

In Fig. [5 we show the region of $\mathrm{w} \leq 1$ (corresponding to $\left.\Omega_{\chi} h^{2} \leq 0.115\right)$ in the $\left(m_{1 / 2}, m_{0}\right)$ plane for $A_{0}=0, \tan \beta=10$, whereas we have chosen $\mu>0$ motivated by $(g-2)_{\mu}$ data. In the CMSSM, the only mechanism which provides a dark matter abundance consistent with the WMAP value, if we require agreement with $(g-2)_{\mu}$ at $3 \sigma$, is stau-neutralino coannihilations [63]. This region is located in a narrow band above the stau-neutralino degeneracy line $\left(m_{\tilde{\tau}}=m_{\chi}\right)$. In addition to this relic abundance constraint, the only effective constraint in this region comes from direct Higgs searches at LEP which excludes low gaugino masses $m_{1 / 2} \lesssim 300 \mathrm{GeV}$. In this figure, the allowed 


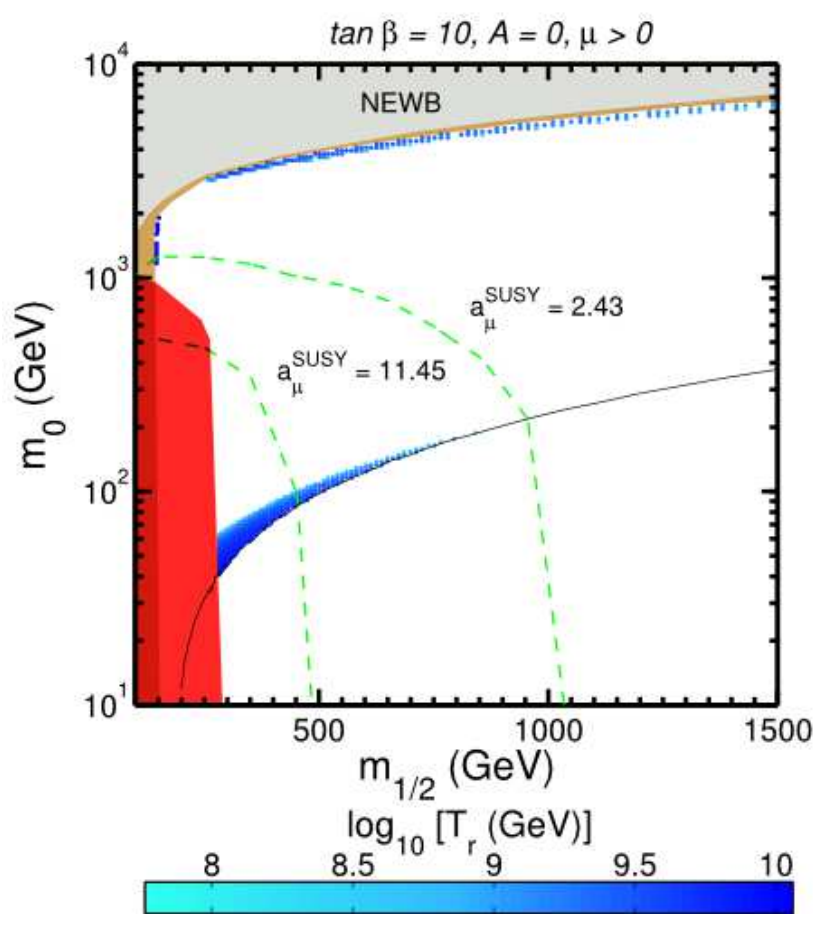

Figure 6: Maximal reheating temperatures in the $m_{1 / 2}-m_{0}$ plane corresponding to the values of $\mathrm{w}$ in Fig. 5 ,

values for $\mathrm{w}$ are shown in different colours. As we can see, in this case, we can only obtain $\mathrm{w}$ between 1 and $\mathcal{O}\left(10^{-1}\right)$. In the region of $m_{\widetilde{\tau}} \leq m_{\chi}$, although the annihilation mechanisms are more efficient than in the neutralino case due to fact that the stau is a charged particle, the lowest $\mathrm{w}$ we can reach is $\mathrm{w}_{\text {min }} \sim 7 \times 10^{-2}$, whereas from Eq. (20) we see that the most conservative bound $Y_{\widetilde{\tau}}<10^{-14}$ from catalyzed nuclear reactions would require $\mathrm{w} \lesssim 2.7 \times 10^{-3}$. Hence, this region of $m_{\tilde{\tau}} \leq m_{\chi}$ is completely excluded for all values of $\Delta M$. If we eliminate the requirement of a non-zero SUSY contribution to $(g-2)_{\mu}$, we obtain two small regions. First, there is a vertical strip at high $m_{0}$ corresponding to a pole in the annihilation $\chi \chi \rightarrow b \bar{b}$ via the lightest Higgs. In this region we can obtain values of $\mathrm{w}$ as low as $\mathrm{w} \simeq 0.01$. Then, there is a long strip, below the non-electroweak symmetry breaking (NEWB) region, at very large $m_{0}$, where the neutralino is a mixed Bino-Higgsino state and the annihilation to $W^{+} W^{-}$is efficient enough to get a right relic abundance. However, in this region we obtain always $\mathrm{w} \gtrsim 0.1$.

Using these allowed values for $\mathrm{w}$ and the corresponding $\omega$ values, we obtain a bound on the mass difference $\Delta M$ in our degenerate gravitino scenario from Figure 2, According to Figure 2, for $\omega \geq 0.03$ the relevant constraint is diffuse gamma ray observations, and this requires $\Delta M \leq 10^{-2}$ $\mathrm{GeV}$. This means that the lifetime of the NLSP (neutralino or gravitino) is longer than the age of the universe. Notice that, in the case of gravitino LSP, the fact that we cannot obtain $\mathrm{w}=\omega<0.1$ implies that this scenario would be completely ruled out by BBN, CMB and diffuse gamma ray observations, unless $\Delta M<10^{-2} \mathrm{GeV}$ and the neutralinos are beginning to decay at present. However, if the 
gravitino is the NLSP, we have two possibilities. The first possibility would be that the observed dark matter abundance is provided completely by the neutralino $\mathrm{w} \simeq 1$, with only a small fraction, at the level of $10^{-3}$, due to gravitinos. This corresponds to $T_{\mathrm{RH}}$ of the order of $10^{7} \mathrm{GeV}$, which is the usual situation in previous studies. The second possibility would be that there is a sizeable fraction of the dark matter due to gravitinos but, again, this would require $\Delta M<10^{-2} \mathrm{GeV}$ corresponding to a very long gravitino lifetime which permits to evade the cosmological bounds. The maximum values of $T_{\mathrm{RH}}$ in these scenarios are shown in Fig. 6. As it can be seen from this figure, values of $T_{\mathrm{RH}}>10^{9} \mathrm{GeV}$ consistent with thermal leptogenesis are accessible in the model, although this is only possible if $\Delta M \leq 10^{-2} \mathrm{GeV}$.

\subsection{Large $\tan \beta=50$}

In Fig. 7, we analyze the case of $\tan \beta=50$. For large values of $\tan \beta$, in the neutralino MSSM LSP region $\left(m_{\chi} \leq m_{\widetilde{\tau}}\right)$, we have different mechanisms to get a correct relic density consistent with WMAP. These mechanisms are i) stau-neutralino coannihilations, ii) "A-pole" region, where the s-channel exchange of the CP-odd Higgs boson, A, can become nearly resonant [64, 65] and iii) the "focus point" or "hyperbolical branch" where a significant higgsino component, enhances its annihilation cross sections into final states containing gauge and/or Higgs bosons [66, 67, 68]. In fact, the focus point region occurs at $m_{0}$ much larger than $m_{1 / 2}$ and therefore multi-TeV scalar masses which implies that $\delta a_{\mu}^{M S S M}$ is well below the lower $2 \sigma$ limit due to SUSY decoupling [69]. However, if we accept $\delta a_{\mu}^{M S S M}$ at the $3 \sigma$ level we can also reach the "focus point" region for $m_{0} \simeq 2 \times 10^{3} \mathrm{GeV}$. Both the neutralino-stau coannihilation band and the "A-pole" region merge for $\tan \beta=50$ as we can see in this figure. This region is cut from the small $m_{1 / 2}$ values by the lower $2 \sigma$ limit of the $B R\left(\bar{B} \rightarrow X_{s} \gamma\right)$ constraint 15 . This constraint limits the minimum possible value of $\mathrm{w}$ obtainable through the "A-pole". Therefore we can only reach $\mathrm{w}_{\min } \sim 0.1$ both in the coannihilation and "A-pole" regions. In the "focus point" region the situation is again similar and we can reach only values $\mathrm{w}_{\min } \sim 0.1-0.01$.

In the case of $m_{\widetilde{\tau}} \leq m_{\chi}$, we can observe an allowed narrow band close to the tachyonic region in which the staus annihilate to $b \bar{b}$ via a pole in the s-channel exchanging a lightest Higgs. This process allows for values of $\mathrm{w}_{\text {min }} \sim 10^{-5}$ which are consistent with the conservative CBBN constraint, $Y_{\widetilde{\tau}}<10^{-14}-10^{-15}$. The broader band at large $m_{1 / 2}$ corresponds to the annihilation into a pair of light Higgses exchanging a light Higgs via a s-channel and here we obtain $\mathrm{w}_{\min } \sim 10^{-3}$ and therefore $Y_{\widetilde{\tau}}<10^{-14}$. On the other hand, we have to keep in mind that if we had imposed the more severe constraint $Y_{\widetilde{\tau}}<10^{-16}$, both regions would be ruled out.

Likewise, with these values for w, we obtain, from Figure 2, the allowed values for the mass difference $\Delta M$. In the neutralino-stau coannihilation or A-pole regions only $\Delta M \leq 10^{-2} \mathrm{GeV}$ are allowed, similarly to the $\tan \beta=10$ case. However, in the region of $m_{\widetilde{\tau}} \leq m_{\chi}$ with values of

\footnotetext{
${ }^{15}$ The reason for this is the destructive interference of the chargino/squark loops which grows with $\tan \beta$ [70]. This effect is enhanced for chargino masses $\leq 100 \mathrm{GeV}$ whereas is never dramatic for masses around 1 $\mathrm{TeV}$ or larger.
} 


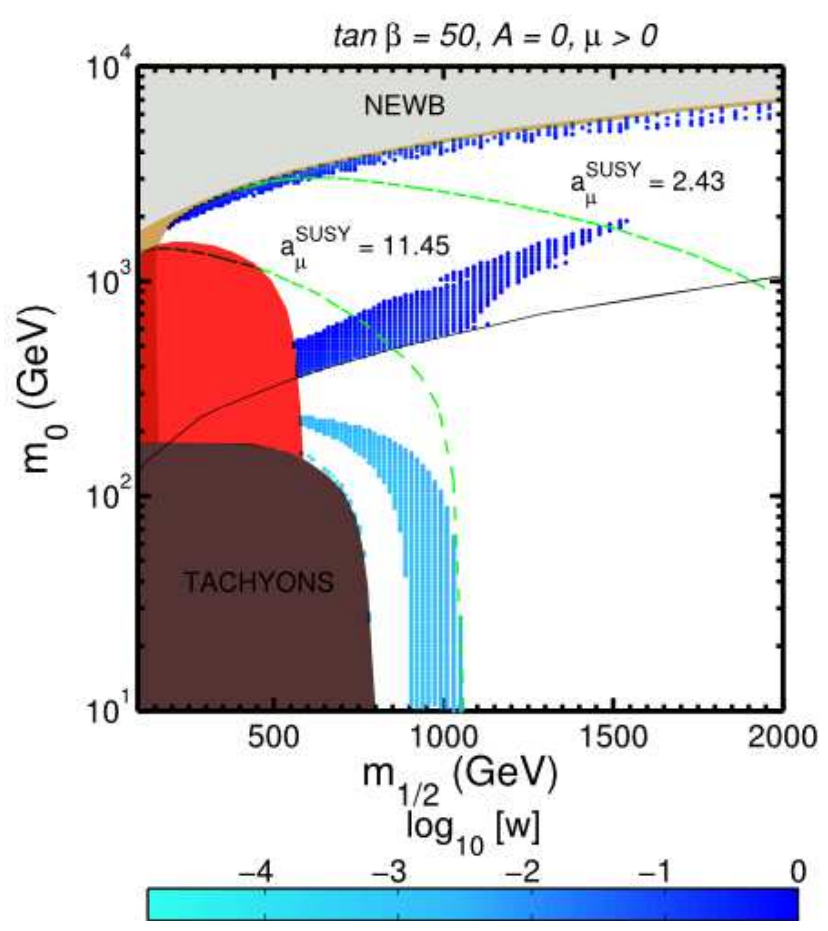

Figure 7: Values of $\mathrm{w}$ in the $m_{1 / 2}-m_{0}$ plane of the CMSSM parameter space for $m_{t}=173.1 \mathrm{GeV}$, $A_{0}=0, \tan \beta=50$ and $\mu>0$. In this case, the red region represents the bound from $\operatorname{BR}(b \rightarrow s \gamma)$ at $2 \sigma$ and there is a new region in dark brown (dark grey) marked "TACHYONS" corresponding to the presence of tachyonic masses. The other colored regions have the same meaning as in the case of $\tan \beta=10$. Regions below the dashed green lines satisfy the $(g-2)_{\mu}$ constraint at the $2 \sigma$ or 3 $\sigma$ level.

$\mathrm{w} \gtrsim 10^{-5}$, we can see from Fig. 4 that values of the mass difference, $90 \mathrm{GeV}>\Delta M \gtrsim 1 \mathrm{GeV}$, would be still allowed for these points 16 .

The maximum values of $T_{\mathrm{RH}}$ are shown in Fig. 8. As we have seen before, both for the gravitino LSP and gravitino NLSP cases $T_{\mathrm{RH}} \propto(1-\mathrm{w})$. The Maximal reheating temperatures for $m_{\chi}<m_{\widetilde{\tau}}$ are always $T_{\mathrm{RH}}>10^{9} \mathrm{GeV}$ if the condition of $\Delta M \lesssim 10^{-2} \mathrm{GeV}$ is satisfied, as in the case of $\tan \beta=10$. However, the region below the stau-neutralino degeneracy line are only allowed in the case of gravitino LSP when the maximal reheating temperatures are again $T_{\mathrm{RH}}>10^{9} \mathrm{GeV}$, although in this case we do not require a tight degeneracy between gravitino and stau.

\subsection{Large $\tan \beta=55$}

In Fig. 9, the case of $\tan \beta=55$ is explored. Similarly to the case of $\tan \beta=50$, in the neutralino MSSM LSP region, the main annihilation mechanisms are the neutralino-stau coannihilation and the "A-pole" resonance, however the "focus point" region is absent in this case. Again the $B R\left(\bar{B} \rightarrow X_{s} \gamma\right)$

\footnotetext{
${ }^{16}$ We are considering only $\Delta M<90 \mathrm{GeV}$ where the hadronic BBN constraints are not efficient.
} 


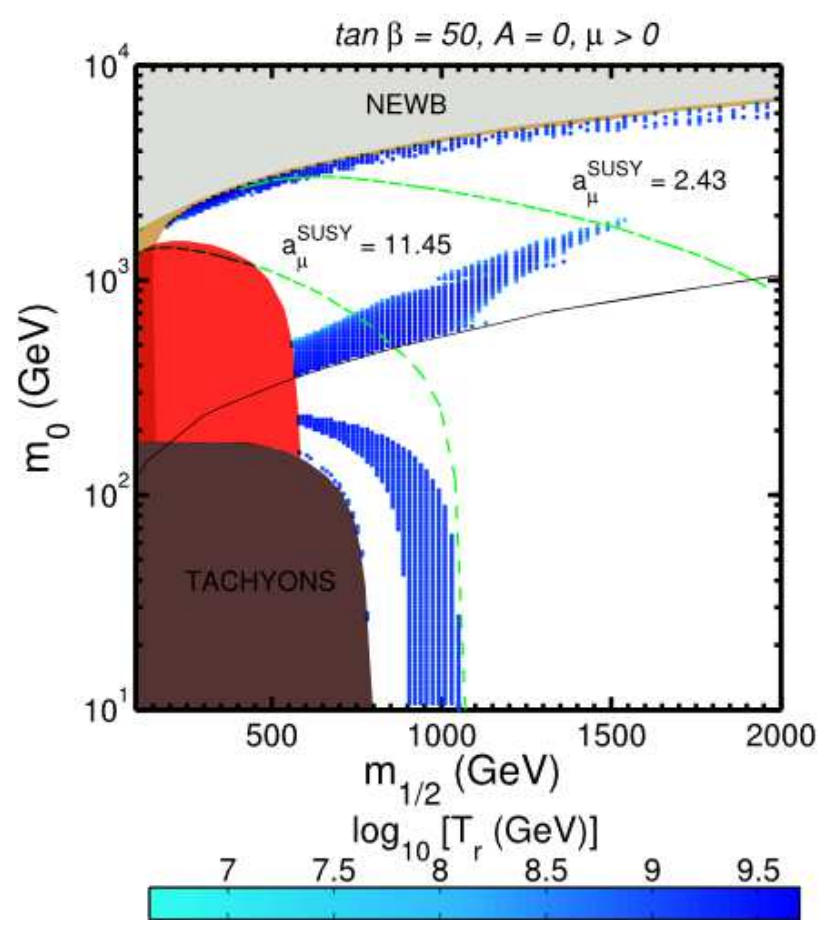

Figure 8: Maximal reheating temperatures in the $m_{1 / 2}-m_{0}$ plane corresponding to the values of $\mathrm{w}$ in Fig. 7 Notice that the region below the stau-neutralino degeneracy line is not allowed in the case of gravitino NLSP.

constraint limits the minimum possible value of w obtainable through the "A-pole". Therefore we can only reach $\mathrm{w}_{\min } \sim 0.1$, both in the coannihilation and "A-pole" regions.

In the case of $m_{\widetilde{\tau}} \leq m_{\chi}$, as for $\tan \beta=50$, staus annihilate through an s-channel exchange of the light Higgs to a pair of light Higgses, although the annihilation to $b \bar{b}$ is suppressed in this case (it does not satisfy the required constraint $\left.Y_{\widetilde{\tau}}<10^{-14}\right)$. The allowed process, $\widetilde{\tau} \widetilde{\tau} \rightarrow h h$, allows for values of $\mathrm{w}_{\text {min }} \sim 6 \times 10^{-4}$ which are consistent with the conservative CBBN constraint for $Y_{\widetilde{\tau}}<10^{-15}$.

Likewise, with these values of $\mathrm{w}$, from Figure 2 we obtain the allowed values for the mass difference $\Delta M$. As before, in the neutralino-stau coannihilation or A-pole regions only $\Delta M \leq 10^{-2}$ $\mathrm{GeV}$ are allowed and in the region of $m_{\tilde{\tau}} \leq m_{\chi}$ mass differences $90 \mathrm{GeV}>\Delta M>2 \mathrm{GeV}$ would be still allowed. The corresponding values of $T_{\mathrm{RH}}$ are shown in Fig. 10. The discussion of section 4.2 applies also in this case.

\section{Conclusions}

Even though they are attractive candidates for cold dark matter, gravitinos are usually a problem in standard cosmology. This is due to the fact that they typically decay at or just after the BBN putting in danger the successful light-elements abundances. Requiring that gravitinos do not conflict with big-bang nucleosynthesis implies that the reheating temperature should be low. In general, there 


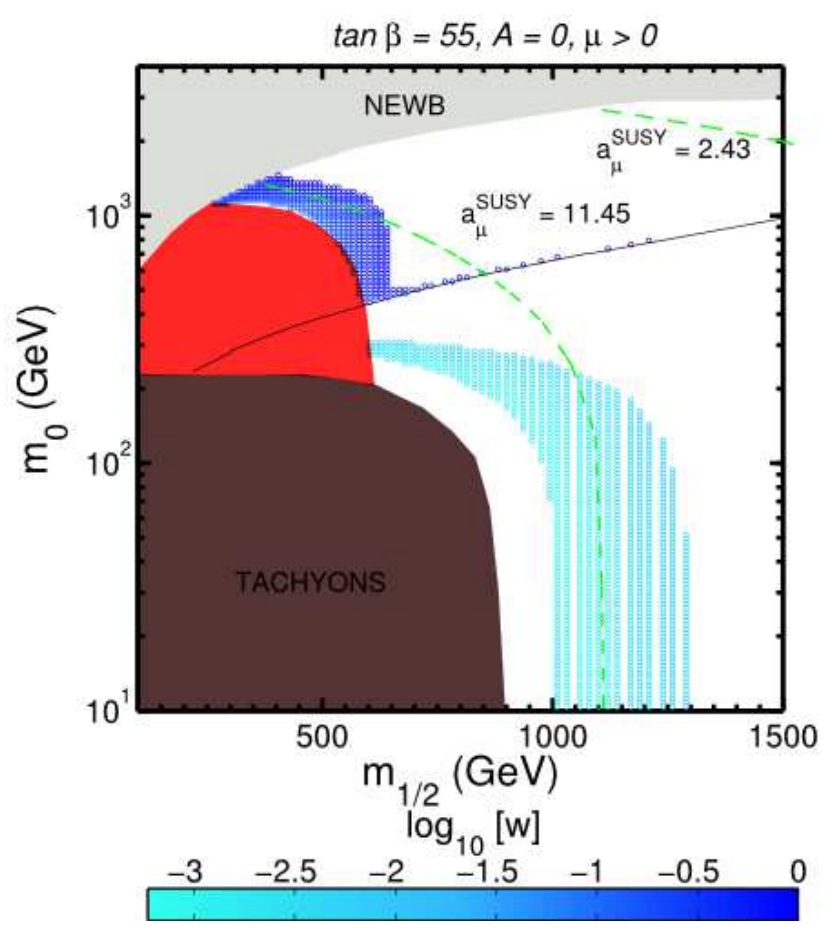

Figure 9: Values of $\mathrm{w}$ in the $m_{1 / 2}-m_{0}$ plane of the CMSSM parameter space for $m_{t}=173.1 \mathrm{GeV}$, $A_{0}=0, \tan \beta=55$ and $\mu>0$. The meaning of the different regions is the same as in Fig. 7 , The regions below the dashed green lines satisfy the $(g-2)_{\mu}$ constraint at the $2 \sigma$ or $3 \sigma$ level.

is no experimental constraint on how low $T_{\mathrm{RH}}$ should be, so in principle a reheating temperature as low as $\mathrm{MeV}$, so to permit BBN, is perfectly allowed. However, successful thermal leptogenesis requires a high reheating temperature $T_{\mathrm{RH}} \gtrsim 2 \times 10^{9} \mathrm{GeV}$.

In this paper, we propose a solution that alleviates this tension by making the gravitino degenerate with the lightest MSSM particle. This has the direct consequence that the injected energy is suppressed, making the decay products less dangerous for BBN. Due to the small mass splitting, the gravitino and the MSSM lightest particle are typically long-lived. We analysed this scenario (the "degenerate gravitino" scenario) by confronting it to cosmological and astrophysical constraints. Since the NLSP decays at or after BBN, we considered in addition to BBN, constraints from CMB spectral distortions and diffuse gamma rays observations. First we performed a model-independent analysis by considering a generic NLSP-LSP degenerate scenario where the NLSP decays through $\mathrm{NLSP} \rightarrow \mathrm{LSP}+X \quad(X=\gamma, \tau)$. Since the final cold dark matter relic density is the sum of both thermal and non-thermal contributions, we required that the total cold dark matter is consistent with cosmological observation. Then, using the results of this analysis, we studied the degenerate gravitino scenario in the context of the CMSSM where three types of spectra arise, they are: gravitino NLSP with neutralino LSP and gravitino LSP with neutralino or stau NLSP. Each of these cases has been analysed in this framework defined by the usual high energy parameters $\left(m_{0}, m_{1 / 2}\right.$, $\left.A_{0}, \tan \beta, \operatorname{sign}(\mu)\right)$ and the gravitino mass $m_{3 / 2}$, and confronted with low-energy observables. We 


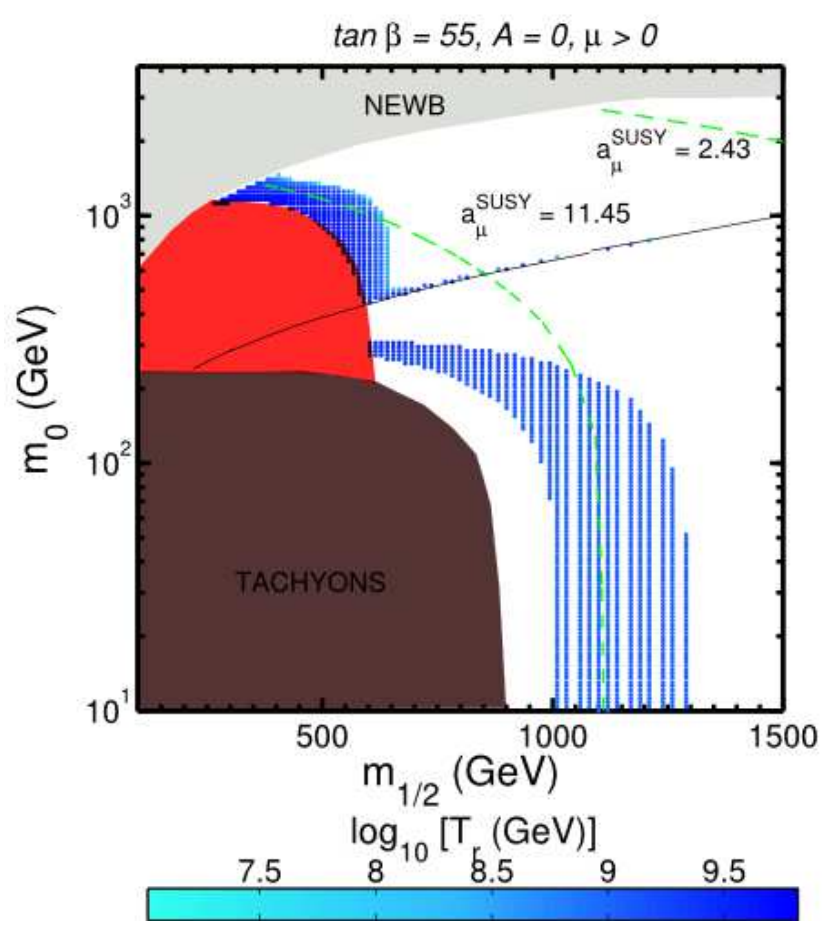

Figure 10: Maximal reheating temperatures in the $m_{1 / 2}-m_{0}$ plane corresponding to the values of $\mathrm{w}$ in Fig. 9. As before the region below the stau-neutralino degeneracy line is not allowed in the case of gravitino NLSP.

find that high reheating temperatures consistent with thermal leptogenesis can be found in all three scenarios if a sizable part of cold dark matter comes from gravitinos produced at reheating. In this case, depending on $\tan \beta$, we are led to regions in the parameter space where the relic density of stau and neutralinos are somewhat suppressed. In general to satisfy all the constraints, the mass splitting between the NLSP and LSP should be $\Delta M \simeq 10^{-2} \mathrm{GeV}$ for the degenerate neutralinogravitino scenario, which implies very long-lived NLSPs which are beginning to decay at present. On the other hand, in the gravitino-stau scenario, splittings in the range $10 \mathrm{GeV} \lesssim \Delta M \lesssim 90 \mathrm{GeV}$ are still consistent with reheating temperatures of the order of $10^{9} \mathrm{GeV}$ if we consider the conservative CBBN constraint $Y_{\mathrm{CBBN}} \leq 10^{-15}$.

Let us comment on the required degeneracy in the "degenerate gravitino" scenario. Although a degeneracy of the order of $\Delta M \simeq 10^{-2} \mathrm{GeV}$ certainly implies a certain amount of fine-tuning, this tuning is only two orders of magnitude stronger than the usual tuning required in the coannihilation or funnel regions to obtain the right relic density in the MSSM. On the other hand, notice also that the fine tuning in our scenario is much softer that the tuning required in other scenarios like inelastic dark matter [71].

Finally, it is also important to consider the phenomenological consequences of this scenario in colliders. In the case of neutralino LSP or NLSP, the only indirect signal of this scenario will be that the relic density of neutralinos inferred from the measurements of supersymmetric masses and 
couplings at LHC, will not match the observed cold dark matter abundance and will be smaller. However, the measurements at direct detection experiments will agree with the cross sections obtained from colliders. On the other hand, for stau NLSP, the collider signatures would be spectacular, as the staus would be completely stable on collider scales and slow charged tracks will appear in the detector [72. Notice that similar signatures can arise in other scenarios like for instance in degenarate neutralino-stau scenario [43] or in gauge-mediation scenarios. However, since the typical stau lifetimes in these scenarios are smaller than $1 \mathrm{sec}$, some of the staus will decay inside the detector. In contrast, stau lifetimes range from $10^{9}-10^{15}$ seconds in our scenario, making it very difficult to observe. Nevertheless, following the analysis of [73], stau lifetimes could be measured at LHC for mass splittings $30 \mathrm{GeV} \lesssim \Delta M \lesssim 90 \mathrm{GeV}$, corresponding to lifetimes $10^{10} \mathrm{sec} \gtrsim \tau_{\widetilde{\tau}} \gtrsim 10^{8}$ sec. In this case, direct detection experiments will give a null results as all the dark matter at present times is made of gravitinos. Therefore, the "degenerate gravitino" scenario will be probed at colliders and direct detection experiments if SUSY is discovered at LHC.

Note added: While completing this work we noticed the preprint 74] where the astrophysical consequences in dark matter halo properties of a neutral long lived decaying-dark matter particle were studied. The required parameters in our "degenerate gravitino" scenario in the case of neutralino LSP or NLSP are such that the constraints of 74 are satisfied.

\section{Acknowledgments}

We thank T. Moroi for useful discussions. O. V. and L. B. acknowledge financial support from spanish MEC and FEDER (EC) under grant FPA2008-02878 and Generalitat Valenciana under the grant PROMETEO/2008/004. O. V was supported in part by European program MRTN-CT-2006035482 "Flavianet". L. B. thanks the Abdus Salam ICTP, the Service de Physique Théorique of ULB Brussels and the CERN-TH division for hospitality during the completion of this work. K.Y. Choi was partly supported by the Korea Research Foundation Grant funded by the Korean Government (KRF-2008-341-C00008) and by the second stage of Brain Korea 21 Project in 2006. The work of R. Ruiz de Austri has been supported in part by MEC (Spain) under grant FPA2007-60323, by Generalitat Valenciana under grant PROMETEO/2008/069 and by the Spanish Consolider-Ingenio 2010 Programme CPAN (CSD2007-00042). The use of the ciclope cluster of the IFT-UAM/CSIC is also acknowledged.

\section{References}

[1] C. Picciotto and M. Pospelov, "Unstable relics as a source of galactic positrons," Phys. Lett. B 605 (2005) 15 arXiv:hep-ph/0402178].

[2] D. Hooper and L. T. Wang, "Evidence for axino dark matter in the galactic bulge," Phys. Rev. D 70 (2004) 063506 [arXiv:hep-ph/0402220]. 
[3] H. Yuksel and M. D. Kistler, "Dark Matter Might Decay... Just Not Today!," Phys. Rev. D 78 (2008) 023502 arXiv:0711.2906 [astro-ph]].

[4] T. Moroi, "Effects of the gravitino on the inflationary universe," arXiv:hep-ph/9503210.

[5] E. Komatsu et al. [WMAP Collaboration], "Five-Year Wilkinson Microwave Anisotropy Probe (WMAP) Observations:Cosmological Interpretation," Astrophys. J. Suppl. 180, 330 (2009) arXiv:0803.0547 [astro-ph]].

[6] K. Jedamzik, K. Y. Choi, L. Roszkowski and R. Ruiz de Austri, "Solving the cosmic lithium problems with gravitino dark matter in the CMSSM," JCAP 0607 (2006) 007 arXiv:hep-ph/0512044.

[7] S. Bailly, K. Jedamzik and G. Moultaka, "Gravitino Dark Matter and the Cosmic Lithium Abundances," Phys. Rev. D 80 (2009) 063509 arXiv:0812.0788 [hep-ph]].

[8] R. H. Cyburt, J. R. Ellis, B. D. Fields and K. A. Olive, "Updated nucleosynthesis constraints on unstable relic particles," Phys. Rev. D 67 (2003) 103521 arXiv:astro-ph/0211258.

[9] K. Jedamzik, "Did something decay, evaporate, or annihilate during big bang nucleosynthesis?," Phys. Rev. D 70 (2004) 063524 [arXiv:astro-ph/0402344].

[10] M. Kawasaki, K. Kohri and T. Moroi, "Hadronic decay of late-decaying particles and big-bang nucleosynthesis," Phys. Lett. B 625 (2005) 7 arXiv:astro-ph/0402490];

M. Kawasaki, K. Kohri and T. Moroi, "Big-bang nucleosynthesis and hadronic decay of longlived massive particles," Phys. Rev. D 71 (2005) 083502 [arXiv:astro-ph/0408426].

[11] K. Jedamzik, "Big bang nucleosynthesis constraints on hadronically and electromagnetically decaying relic neutral particles," Phys. Rev. D 74 (2006) 103509 arXiv:hep-ph/0604251].

[12] J. R. Ellis, J. E. Kim and D. V. Nanopoulos, "Cosmological Gravitino Regeneration And Decay," Phys. Lett. B 145, 181 (1984).

[13] J. L. Feng, S. Su and F. Takayama, "Supergravity with a gravitino LSP," Phys. Rev. D 70 (2004) 075019 arXiv:hep-ph/0404231.

[14] J. R. Ellis, K. A. Olive, Y. Santoso and V. C. Spanos, "Gravitino dark matter in the CMSSM," Phys. Lett. B 588 (2004) 7 arXiv:hep-ph/0312262.

[15] L. Roszkowski, R. Ruiz de Austri and K. Y. Choi, "Gravitino dark matter in the CMSSM and implications for leptogenesis and the LHC," JHEP 0508 (2005) 080 arXiv:hep-ph/0408227.

[16] K. Kohri, T. Moroi and A. Yotsuyanagi, "Big-bang nucleosynthesis with unstable gravitino and upper bound on the reheating temperature," Phys. Rev. D 73 (2006) 123511 arXiv:hep-ph/0507245. 
[17] D. G. Cerdeno, K. Y. Choi, K. Jedamzik, L. Roszkowski and R. Ruiz de Austri, "Gravitino dark matter in the CMSSM with improved constraints from BBN," JCAP 0606 (2006) 005 arXiv:hep-ph/0509275].

[18] F. D. Steffen, "Gravitino dark matter and cosmological constraints," JCAP 0609 (2006) 001 arXiv:hep-ph/0605306.

[19] J. Pradler and F. D. Steffen, "Thermal Gravitino Production and Collider Tests of Leptogenesis," Phys. Rev. D 75 (2007) 023509 arXiv:hep-ph/0608344.

[20] M. Kawasaki, K. Kohri, T. Moroi and A. Yotsuyanagi, "Big-Bang Nucleosynthesis and Gravitino," Phys. Rev. D 78 (2008) 065011 arXiv:0804.3745 [hep-ph]].

[21] S. Bailly, K. Y. Choi, K. Jedamzik and L. Roszkowski, "A Re-analysis of Gravitino Dark Matter in the Constrained MSSM," JHEP 0905 (2009) 103 arXiv:0903.3974 [hep-ph]].

[22] J. A. R. Cembranos, J. L. Feng and L. E. Strigari, "Resolving Cosmic Gamma Ray Anomalies with Dark Matter Decaying Now," Phys. Rev. Lett. 99 (2007) 191301 arXiv:0704.1658 [astro$\mathrm{ph}]$.

[23] D. J. Fixsen, E. S. Cheng, J. M. Gales, J. C. Mather, R. A. Shafer and E. L. Wright, "The Cosmic Microwave Background Spectrum from the Full COBE/FIRAS Data Set," Astrophys. J. 473 (1996) 576 arXiv:astro-ph/9605054.

[24] W. Hu and J. Silk, "Thermalization constraints and spectral distortions for massive unstable relic particles," Phys. Rev. Lett. 70 (1993) 2661.

[25] K. Hagiwara et al. [Particle Data Group], "Review of particle physics," Phys. Rev. D 66 (2002) 010001.

[26] M. Pospelov, "Particle physics catalysis of thermal big bang nucleosynthesis," Phys. Rev. Lett. 98 (2007) 231301 arXiv:hep-ph/0605215;

K. Kohri and F. Takayama, "Big Bang Nucleosynthesis with Long Lived Charged Massive Particles," Phys. Rev. D 76 (2007) 063507 arXiv:hep-ph/0605243;

M. Kaplinghat and A. Rajaraman, "Big Bang Nucleosynthesis with Bound States of Long-lived Charged Particles," Phys. Rev. D 74 (2006) 103004 arXiv:astro-ph/0606209.

[27] K. Hamaguchi, T. Hatsuda, M. Kamimura, Y. Kino and T. T. Yanagida, "Stau-catalyzed Li-6 production in big-bang nucleosynthesis," Phys. Lett. B 650 (2007) 268 arXiv:hep-ph/0702274.

[28] J. Pradler and F. D. Steffen, "Implications of Catalyzed BBN in the CMSSM with Gravitino Dark Matter," Phys. Lett. B 666 (2008) 181 [arXiv:0710.2213 [hep-ph]].

[29] K. Jedamzik, "Bounds on long-lived charged massive particles from Big Bang nucleosynthesis," JCAP 0803 (2008) 008 arXiv:0710.5153 [hep-ph]]. 
[30] K. Jedamzik and M. Pospelov, "Big Bang Nucleosynthesis and Particle Dark Matter," arXiv:0906.2087 [hep-ph].

[31] C. F. Berger, L. Covi, S. Kraml and F. Palorini, "The number density of a charged relic," JCAP 0810 (2008) 005 [arXiv:0807.0211 [hep-ph]].

[32] M. Ratz, K. Schmidt-Hoberg and M. W. Winkler, "A note on the primordial abundance of stau NLSPs," JCAP 0810 (2008) 026 arXiv:0808.0829 [hep-ph]].

[33] J. Pradler and F. D. Steffen, "Thermal relic abundances of long-lived staus," Nucl. Phys. B 809 (2009) 318 arXiv:0808.2462 [hep-ph]].

[34] H. P. Nilles, M. Peloso and L. Sorbo, "Nonthermal production of gravitinos and inflatinos," Phys. Rev. Lett. 87 (2001) 051302 arXiv:hep-ph/0102264.

[35] M. Bolz, A. Brandenburg and W. Buchmuller, "Thermal Production of Gravitinos," Nucl. Phys. B 606 (2001) 518 [Erratum-ibid. B 790 (2008) 336] arXiv:hep-ph/0012052].

[36] V. S. Rychkov and A. Strumia, "Thermal production of gravitinos," Phys. Rev. D 75 (2007) 075011 arXiv:hep-ph/0701104].

[37] S. Davidson and A. Ibarra, "A lower bound on the right-handed neutrino mass from leptogenesis," Phys. Lett. B 535, 25 (2002) arXiv:hep-ph/0202239.

[38] G. F. Giudice, A. Notari, M. Raidal, A. Riotto and A. Strumia, "Towards a complete theory of thermal leptogenesis in the SM and MSSM," Nucl. Phys. B 685 (2004) 89 arXiv:hep-ph/0310123.

[39] S. Antusch and A. M. Teixeira, "Towards constraints on the SUSY seesaw from flavourdependent leptogenesis," JCAP 0702 (2007) 024 arXiv:hep-ph/0611232.

[40] M. Olechowski, S. Pokorski, K. Turzynski and J. D. Wells, "Reheating Temperature and Gauge Mediation Models of Supersymmetry Breaking," arXiv:0908.2502 [hep-ph].

[41] C. Arina and N. Fornengo, "Sneutrino cold dark matter, a new analysis: Relic abundance and detection rates," JHEP 0711 (2007) 029 arXiv:0709.4477 [hep-ph]].

[42] A. Masiero, S. K. Vempati and O. Vives, "Massive neutrinos and flavour violation," New J. Phys. 6 (2004) 202 arXiv:hep-ph/0407325.

[43] S. Kaneko, J. Sato, T. Shimomura, O. Vives and M. Yamanaka, "Measuring Lepton Flavour Violation at LHC with Long-Lived Slepton in the Coannihilation Region," Phys. Rev. D 78 (2008) 116013 arXiv:0811.0703 [hep-ph]].

[44] T. Yamagata, Y. Takamori and H. Utsunomiya, "Search for anomalously heavy hydrogen in deep sea water at 4000-m," Phys. Rev. D 47, 1231 (1993). 
[45] H. P. Nilles, Supersymmetry, Supergravity And Particle Physics, Phys. Rept. 110 (1984) 1.

[46] G. L. Kane, C. F. Kolda, L. Roszkowski and J. D. Wells, "Study of constrained minimal supersymmetry", Phys. Rev. D 49 (1994) 6173 hep-ph/9312272.

[47] K. Y. Choi, L. Roszkowski and R. Ruiz de Austri, "Determining Reheating Temperature at Colliders with Axino or Gravitino Dark Matter," JHEP 0804 (2008) 016 arXiv:0710.3349 [hep-ph]].

[48] J. P. Miller, E. de Rafael and B. L. Roberts, "Muon g-2: Review of Theory and Experiment," Rept. Prog. Phys. 70, 795 (2007) arXiv:hep-ph/0703049].

[49] Heavy Flavor Averaging Group (HFAG) (E. Barberio et al.), "Averages of b-hadron properties at the end of 2007", arXiv:0808.1297 [hep-ex].

[50] J. Dunkley et al. [WMAP Collaboration], "Five-Year Wilkinson Microwave Anisotropy Probe (WMAP) Observations: Likelihoods and Parameters from the WMAP data," Astrophys. J. Suppl. 180, 306 (2009) arXiv:0803.0586 [astro-ph]].

[51] R. Barate et al. [LEP Working Group for Higgs boson searches and ALEPH Collaboration], "Search for the standard model Higgs boson at LEP," Phys. Lett. B 565, 61 (2003) arXiv:hep-ex/0306033.

[52] G. Belanger, F. Boudjema, A. Pukhov and A. Semenov, "MicrOMEGAs: A program for calculating the relic density in the MSSM," Comput. Phys. Commun. 149 (2002) 103 hep-ph/0112278; "MicrOMEGAs: Version 1.3," Comput. Phys. Commun. 174, 577 (2006) hep-ph/0405253.

[53] A. Djouadi, J. L. Kneur and G. Moultaka, "SuSpect: A Fortran code for the supersymmetric and Higgs particle spectrum in the MSSM," Comput. Phys. Commun. 176, 426 (2007) arXiv:hep-ph/0211331.

[54] A. Djouadi, M. Drees and J. L. Kneur, "Constraints on the minimal supergravity model and prospects for SUSY particle production at future linear $e^{+} e^{-}$colliders," JHEP 0108, 055 (2001) arXiv:hep-ph/0107316.

[55] G. Degrassi, P. Gambino and P. Slavich, "SusyBSG: a fortran code for BR[B $\rightarrow$ Xs gamma] in the MSSM with Minimal Flavor Violation," Comput. Phys. Commun. 179 (2008) 759 arXiv:0712.3265 [hep-ph]].

[56] G. Degrassi, P. Gambino and P. Slavich, "QCD corrections to radiative B decays in the MSSM with minimal flavor violation," Phys. Lett. B 635 (2006) 335 arXiv:hep-ph/0601135.

[57] G. D'Ambrosio, G. F. Giudice, G. Isidori and A. Strumia, "Minimal flavour violation: An effective field theory approach," Nucl. Phys. B 645, 155 (2002) arXiv:hep-ph/0207036. 
[58] S. Heinemeyer, D. Stockinger and G. Weiglein, "Electroweak and supersymmetric two-loop corrections to (g-2)(mu)," Nucl. Phys. B 699, 103 (2004) arXiv:hep-ph/0405255.

[59] S. Marchetti, S. Mertens, U. Nierste and D. Stockinger, "Tan(beta)-enhanced supersymmetric corrections to the anomalous magnetic moment of the muon," Phys. Rev. D 79 (2009) 013010 arXiv:0808.1530 [hep-ph]].

[60] P. Skands et al., "SUSY Les Houches Accord: Interfacing SUSY Spectrum Calculators, Decay Packages, and Event Generators," JHEP 0407 (2004) 036 arXiv:hep-ph/0311123.

[61] B. C. Allanach, A. Djouadi, J. L. Kneur, W. Porod and P. Slavich, "Precise determination of the neutral Higgs boson masses in the MSSM," JHEP 0409 (2004) 044 arXiv:hep-ph/0406166.

[62] [Tevatron Electroweak Working Group and CDF Collaboration and D0 Collab], "Combination of CDF and D0 Results on the Mass of the Top Quark," arXiv:0903.2503 [hep-ex].

[63] T. Nihei, L. Roszkowski and R. Ruiz de Austri, "Exact Cross Sections for the Neutralino-Slepton Coannihilation," JHEP 0207, 024 (2002) arXiv:hep-ph/0206266.

[64] J. R. Ellis, T. Falk, G. Ganis, K. A. Olive and M. Srednicki, "The CMSSM Parameter Space at Large tan beta," Phys. Lett. B 510, 236 (2001) arXiv:hep-ph/0102098.

[65] L. Roszkowski, R. Ruiz de Austri and T. Nihei, "New cosmological and experimental constraints on the CMSSM," JHEP 0108, 024 (2001) arXiv:hep-ph/0106334.

[66] K. L. Chan, U. Chattopadhyay and P. Nath, "Naturalness, weak scale supersymmetry and the prospect for the observation of supersymmetry at the Tevatron and at the LHC," Phys. Rev. D 58, 096004 (1998) arXiv:hep-ph/9710473.

[67] J. L. Feng, K. T. Matchev and T. Moroi, "Multi-TeV scalars are natural in minimal supergravity," Phys. Rev. Lett. 84, 2322 (2000) arXiv:hep-ph/9908309].

[68] J. L. Feng, K. T. Matchev and T. Moroi, "Focus points and naturalness in supersymmetry," Phys. Rev. D 61, 075005 (2000) arXiv:hep-ph/9909334.

[69] T. Moroi, "The Muon Anomalous Magnetic Dipole Moment in the Minimal Supersymmetric Standard Model," Phys. Rev. D 53, 6565 (1996) [Erratum-ibid. D 56, 4424 (1997)] arXiv:hep-ph/9512396.

[70] G. Degrassi, P. Gambino and G. F. Giudice, "B $\rightarrow \mathrm{X} / \mathrm{s}$ gamma in supersymmetry: Large contributions beyond the leading order," JHEP 0012, 009 (2000) arXiv:hep-ph/0009337.

[71] D. Tucker-Smith and N. Weiner, "Inelastic dark matter," Phys. Rev. D 64 (2001) 043502 arXiv:hep-ph/0101138. 
[72] S. Tarem, and S. Bressler, E. Duchovni and L. Levinson, "Can ATLAS avoid missing the long lived stau?", Atlas note ATL-PHYS-PUB-2005-022, 2005;

S. Tarem, S. Bressler, H. Nomoto and A. Dimattia; "Trigger and Reconstruction for a heavy long lived charged particles with the ATLAS detector", Atlas note ATL-PHYS-PUB-2008-001, 2008 ;

J. R. Ellis, A. R. Raklev and O. K. Oye, "Measuring massive metastable charged particles with ATLAS RPC timing information," Atlas note ATL-PHYS-PUB-2007-016, 2007;

K. Ishiwata, T. Ito and T. Moroi, "Long-Lived Unstable Superparticles at the LHC," Phys. Lett. B 669 (2008) 28 [arXiv:0807.0975 [hep-ph]];

[73] S. Asai, K. Hamaguchi and S. Shirai, "Stop and Decay of Long-lived Charged Massive Particles at the LHC detectors," Phys. Rev. Lett. 103, 141803 (2009) arXiv:0902.3754 [hep-ph]].

[74] A. H. G. Peter, "Mapping the allowed parameter space for decaying dark matter models," arXiv:1001.3870. 